\title{
La traduction du patrimoine: ajout ou perte dans le transfert interculturel ? (Le cas de la traduction du site de la Bibliothèque d'Alexandrie « Mémoire de l’Egypte Moderne ذاكرة مصر الحديثة "
}

\section{Dr. Rim Hafez ELBAKARY*}

\section{Résumé :}

La traduction du Patrimoine se présente comme un fil à retordre pour tout traducteur, même pour le plus chevronné, du fait que toute traduction erronée pourrait biaiser la réception d'un pan entier d'une culture par une autre et générer des incompréhensions, des différends, parfois même des conflits au niveau international.

C'est en faisant la traduction du site de la Bibliothèque d'Alexandrie, intitulé : «Mémoire de l'Égypte Moderne » (MoME)/ذاكرةمصر.الحديثة, qui est une bibliothèque numérique en ligne documentant l'histoire de l'Égypte à partir du règne de Méhémet Ali en 1805 jusqu'au mandat du président Sadate en 1981, que nous nous sommes rendue compte des conséquences que peut entraîner toute impropriété dans la traduction du patrimoine culturel et historique d'un pays en général, de l’Egypte en particulier.

En fait, la traduction de ce site a été réalisée dans le cadre d'un projet mené conjointement entre la Filière des Langues Appliquées de la Faculté de Lettres, où nous travaillons, et le Centre International des Sciences de I'Information et le Département des Projets Spéciaux de la Bibliothèque d'Alexandrie. Ce projet de traduction de La Mémoire de l'Egypte Moderne (MoME) a été proposé en tant que projet de fin d'études aux étudiants de la Filière

Dans le travail que nous avons supervisé, nous avons donc d’abord relevé et classé les impropriétés de traduction commises par les étudiants. Celles-ci étaient tantôt considérées comme des erreurs graves dues à une recherche non-approfondie de la part des étudiants; tantôt considérées comme un choix non-fondé dû à une confusion entre les différents sens d'un mot et les différents contextes où ce mot devait être utilisé. Nous avons alors mené un travail de recherche documentaire dans lequel nous avons contribué et guidé les étudiants. Nous avons ensuite discuté avec eux ces impropriétés ou ces choix traductionnels, soit pour les convaincre de la fausse piste dans laquelle ils se sont engagés, soit pour accepter leur point de vue s'il est convaincant et repose sur une recherche approfondie. Ainsi, nous avons pu aboutir à la traduction la plus adéquate que nous exposerons dans cette étude.

Mots clés : Traduction du Patrimoine-impropriétés et erreurs-choix traductionnels.

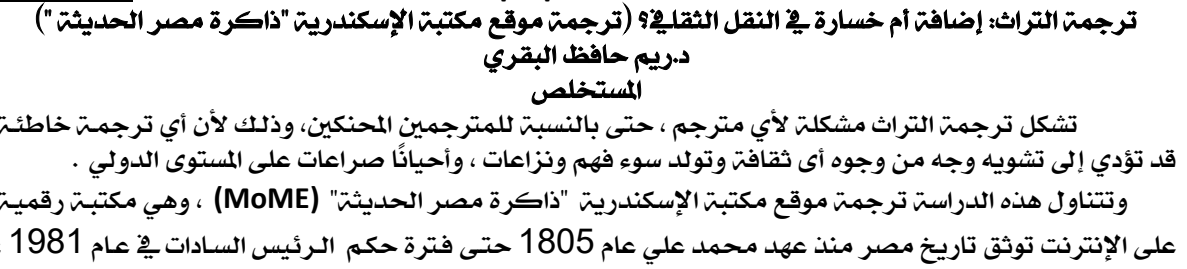

- Maitre de conferences- Filière des Langues Appliquées- Faculté des LettresUniversité d'Alexandrie 


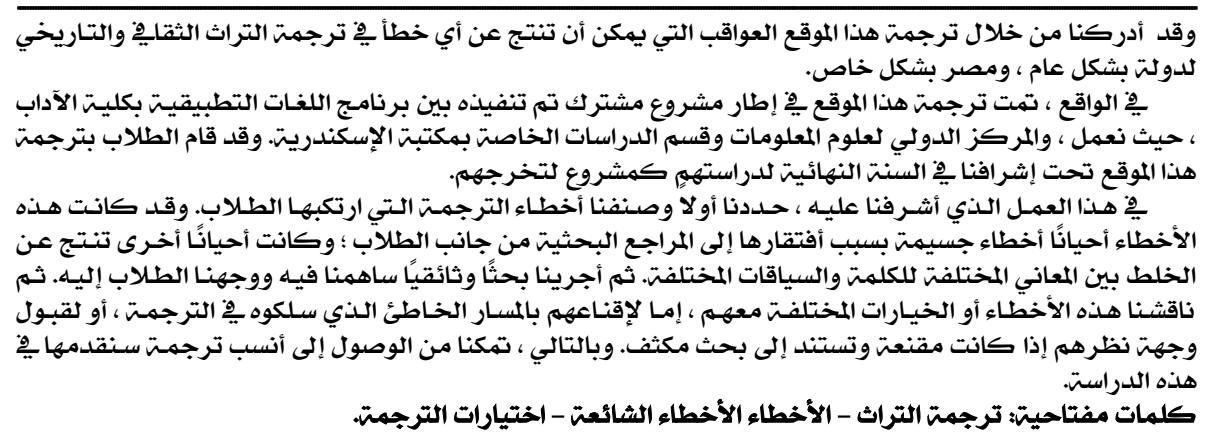

- Introduction :

«Comprendre un texte c'est faire appel à une compétence linguistique et, simultanément, à un savoir encyclopédique $»^{1}$

De tout temps, le traducteur a joué un rôle clé en tant que médiateur entre langues et cultures. Transmettre correctement et objectivement le message initial de l'énonciateur ou de la culture source représente un défi considérable, voire un fil à retordre pour tout traducteur, même pour le plus chevronné, du fait que toute traduction erronée pourrait biaiser la réception d'un pan entier d'une culture par une autre et générer des incompréhensions, des différends, parfois même des conflits au niveau international. Alors quel serait le cas s'il s'agit de la traduction du patrimoine ? En effet, «si le patrimoine se décline en objets, en monuments, en forêts, en richesses animale et végétale, en traditions et récits, il se dit surtout en «mots $»^{2}$.

Pour pouvoir effectuer une traduction fidèle à l'original, et éviter toute inexactitude linguistique, culturelle ou historique dans la traduction du patrimoine, le traducteur doit donc se munir de plusieurs outils et être capable de mobiliser des connaissances approfondies de la culture source. Ainsi le traducteur doit-il se montrer très prudent et très informé lorsqu'il traduit des textes d'importance capitale, comme

${ }^{1}$ Marianne LEDERER, La traduction aujourd'hui. Le modèle interprétatif, Hachette Paris, 1994, p.32.

${ }^{2}$ https://www.les-treilles.com/les-intraduisibles-du-patrimoine

Consulté le 20 septembre 2018. 
ceux du Patrimoine. Il ne faut pas oublier aussi que l'interprétation du traducteur joue un rôle fondamental dans sa traduction. Il doit donc être certain de traduire ce qui a vraiment voulu être dit, s'informer des différences entre les langues et des différences de cultures.

C'est en faisant la traduction du site de la Bibliothèque d'Alexandrie, intitulé : «Mémoire de l'Égypte Moderne »

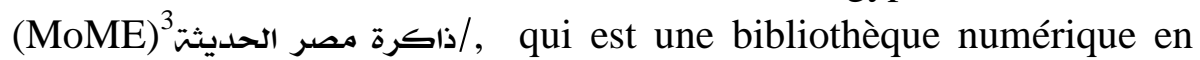
ligne documentant l'histoire de l'Égypte à partir du règne de Méhémet Ali en 1805 jusqu'au mandat du président Sadate en 1981, que nous nous sommes rendue compte des conséquences que peut entraîner toute impropriété dans la traduction du patrimoine culturel et historique d'un pays en général, de l'Egypte en particulier.

En fait, la traduction de ce site a été réalisée dans le cadre d'un projet mené conjointement entre la Filière des Langues Appliquées de la Faculté de Lettres, où nous travaillons, et le Centre International des Sciences de l'Information et le Département des Projets Spéciaux de la Bibliothèque d'Alexandrie. Ce projet de traduction de La Mémoire de l'Egypte Moderne (MoME) a été proposé en tant que projet de fin d'études aux étudiants de la Filière de la promotion 2017. Le projet, qui a duré presqu'un an, consistait à traduire tout le contenu du site, à le soumettre à un comité de révision composé de professeurs de traduction qui encadraient les étudiants de la Filière des Langues Appliquées dans leur projet, et enfin à le remettre au département concerné de la bibliothèque pour effectuer les démarches nécessaires à la publication du contenu du site en langue française. Or, le projet n'a malheureusement pas vu le jour jusqu'à présent pour des raisons de financement et de disponibilité des réviseurs.

Le contenu du site a été donc équitablement divisé entre les étudiants de la quatrième année, de façon à ce que chaque groupe d'étudiants, composé de cinq étudiants au maximum, soit encadré par un professeur dont le rôle, est d'assurer le bon déroulement du projet et de réviser le texte traduit afin d'apporter les modifications nécessaires.

${ }^{3}$ http://modernegypt.bibalex.org/collections/home/default.aspx Dernière consultation le 03/08/2018 
Dans le travail que nous avons supervisé, nous avons d'abord relevé et classé les impropriétés de traduction commises par les étudiants. Celles-ci étaient tantôt considérées comme des erreurs graves dues à une recherche non-approfondie de la part des étudiants ; tantôt considérées comme un choix non-fondé dû à une confusion entre les différents sens d'un mot et les différents contextes où ce mot devait être utilisé. Nous avons alors mené un travail de recherche documentaire dans lequel nous avons contribué et guidé les étudiants. Nous avons ensuite discuté avec eux ces impropriétés ou ces choix traductionnels, soit pour les convaincre de la fausse piste dans laquelle ils se sont engagés, soit pour accepter leur point de vue s'il est convaincant et repose sur une recherche approfondie . Ainsi, nous avons pu aboutir à la traduction la plus adéquate que nous exposerons dans les quelques pages suivantes.

Dans cette étude, basée sur un outil visuel informatif et authentique de l'histoire de l'Égypte qu'est le site «Mémoire de l'Égypte Moderne ", nous avons donc essayé à partir d'exemples concrets relevés tout au long du travail mené, de répondre à quelques interrogations à savoir entre autres: À partir de quel degré d'éloignement du texte original peut-on considérer qu'il y a erreur ? La traduction qui pourrait être considérée comme acceptable à une époque peut-elle tomber en disgrâce aux siècles suivants ? Quels types d'impropriétés de traduction pourraient mener à des conséquences estimées comme désastreuses?

Toutes ces interrogations, auxquelles nous essayerons de répondre dans notre étude, nous mèneront à reconsidérer la traduction du patrimoine d'un point de vue historique et contextuel et voir comment l'erreur ou l'impropriété dans la traduction du patrimoine peut entraver l'exactitude du transfert interculturel.

\section{Contexte et importance de l'étude :}

Le contexte sur lequel porte notre étude est un projet intitulé $L a$ Mémoire de l'Egypte Moderne (MoME) conjointement mené par le Centre International des Sciences de l'Information et le Département des Projets Spéciaux (ISIS) de la BA. Il s'agit d'une bibliothèque numérique portant sur l'histoire de l'Egypte moderne au cours des deux derniers siècles, allant de l'époque de Méhémet Ali jusqu'au mandat du président Sadate en 1981. Cette documentation a été 
réalisée grâce à des milliers d'articles, de photos, de documents, d'un matériel audio-visuel assez riche, de plans, de cartes géographiques ainsi que d'autres supports. Ces supports sont accessibles à la fois en ligne via le site web du projet ${ }^{4}$, et hors ligne, via la revue trimestrielle publiée par le Département des Projets Spéciaux, la Mémoire de l'Egypte'.

L'importance de la réalisation de ce projet repose sur plusieurs raisons majeures: tout d'abord, diffuser la culture et l'histoire égyptiennes dans le monde francophone et sur la scène internationale ; et contribuer ainsi à la création d'un contenu numérique traduit portant sur la culture et l'histoire égyptiennes, vu la pénurie et parfois la nonfiabilité des œuvres traduites sur Internet dans ce domaine. Ensuite, faciliter l'accès à ce type d'information pour ceux qui préfèrent la lecture en ligne surtout les jeunes vivant à l'étranger qui méconnaissent l'histoire et la culture de leur pays et dont la lecture en ligne leur est préférable plus que la lecture de tout document imprimé. Enfin, proposer de nouvelles approches et astuces de traduction qui rapprocheront les points de vue, française et égyptienne, dans le domaine de «l'Egyptologie moderne », et présenter un travail qui sera un point de départ pour d'autres chercheurs et traducteurs francophones intéressés par un domaine jusqu'à lors difficile à appréhender en traduction, celui de l'Egyptologie moderne. Bref, renouer, en tant que traductrice égyptienne, les liens perdus, c'est-àdire compenser par une traduction correcte et documentée, les décalages qu'imposent à leurs lecteurs des notions, des informations, des concepts dits anciens.

\footnotetext{
${ }^{4}$ http://modernegypt.bibalex.org/collections/home/default.aspx

Dernière consultation le $03 / 08 / 2018$

${ }^{5} \mathrm{http} / / / \mathrm{www} \cdot$ bibalex.org/fr/project/details?documentid=1315\&keywords $=$ Dernière consultation le 03/08/2018
} 


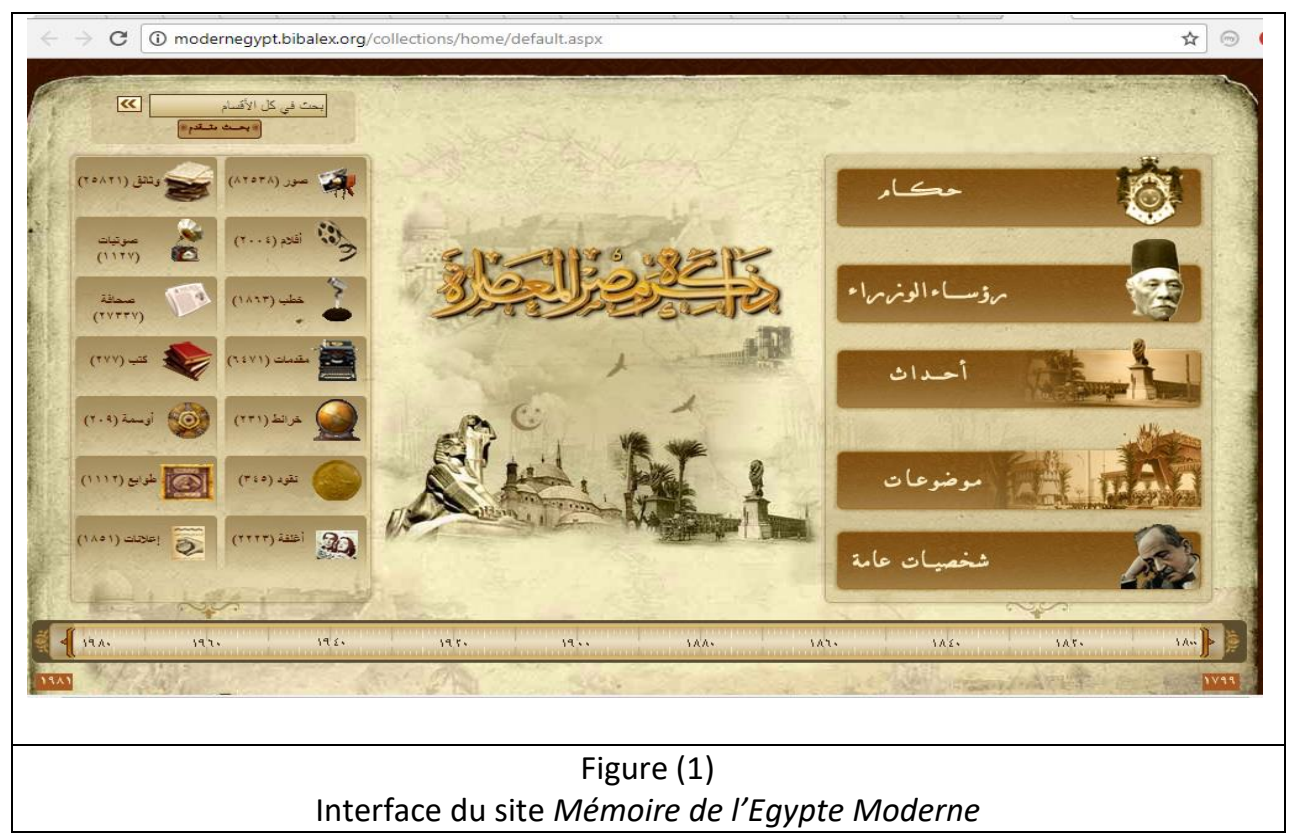

\section{Corpus de l'étude:}

Le corpus, se trouvant sur le site, est constitué de deux grands volets: un premier volet, comprenant le contenu essentiel de la Mémoire de l'Egypte Moderne, et se composant de cinq grandes rubriques; un second volet, comprenant les archives, et se composant de 14 petites rubriques.

Le premier groupe de textes (Figue 2), dans le premier volet, est classé sous une rubrique intitulée «Souverains", et regroupe des légendes retraçant les mandats des différents rois et chefs d'états qui ont gouverné l'Egypte depuis Méhémet Ali jusqu'à l'époque du président Sadat, à savoir:

- L'époque du déclin du règne du Roi Farouk et le coup d'état de 1952.

- L'époque de Muhamad Najib, le premier président de la République.

- L'époque du Président Sadate. 


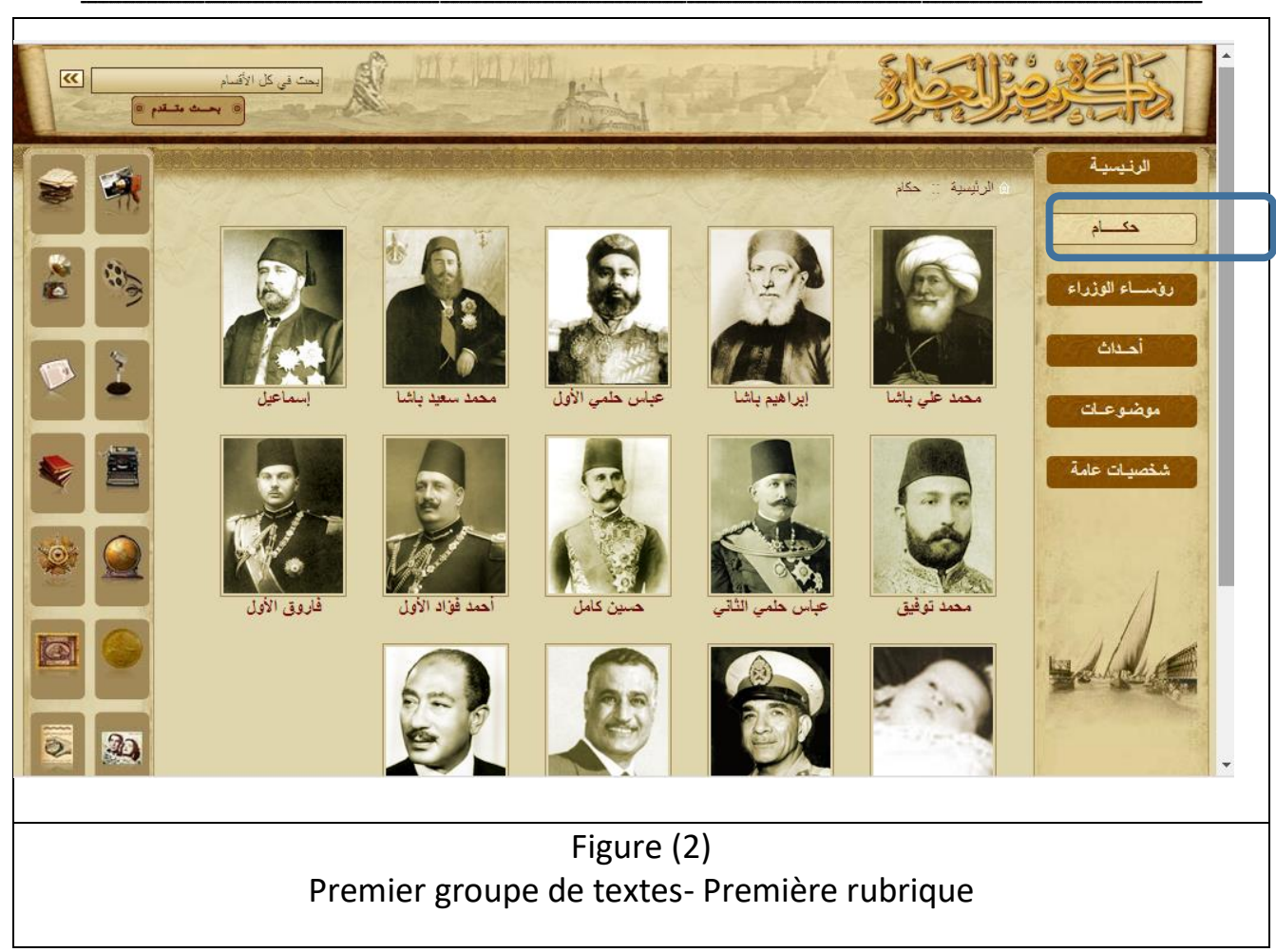

Le deuxième groupe (Figure 3$)^{6}$, appartenant toujours au premier volet et classé sous la rubrique «premiers ministres » est une collecte de textes présentant différentes biographies d'un nombre de premiers ministres au cours de l'histoire de l'Égypte moderne, à savoir:

- Les mandats d'Hassan Sabri Pacha (du 27 / 06 / 1940 jusqu'à 14 / 11 / 1940),

- Les mandats d'Husayn Serry Pacha (du 15/11/1940 jusqu'à 31/07/1941 - du 02/07/1952 jusqu'à 22/07/1952),

- Les mandats d'Ahmed Maher Pacha (du $08 / 10 / 1944$ jusqu'à $15 / 01 / 1945$ - du $15 / 01 / 1945$ jusqu'à $24 / 02 /$ 1945).

${ }^{6} \mathrm{Cf}$., infra p.7 pour la figure (3) 


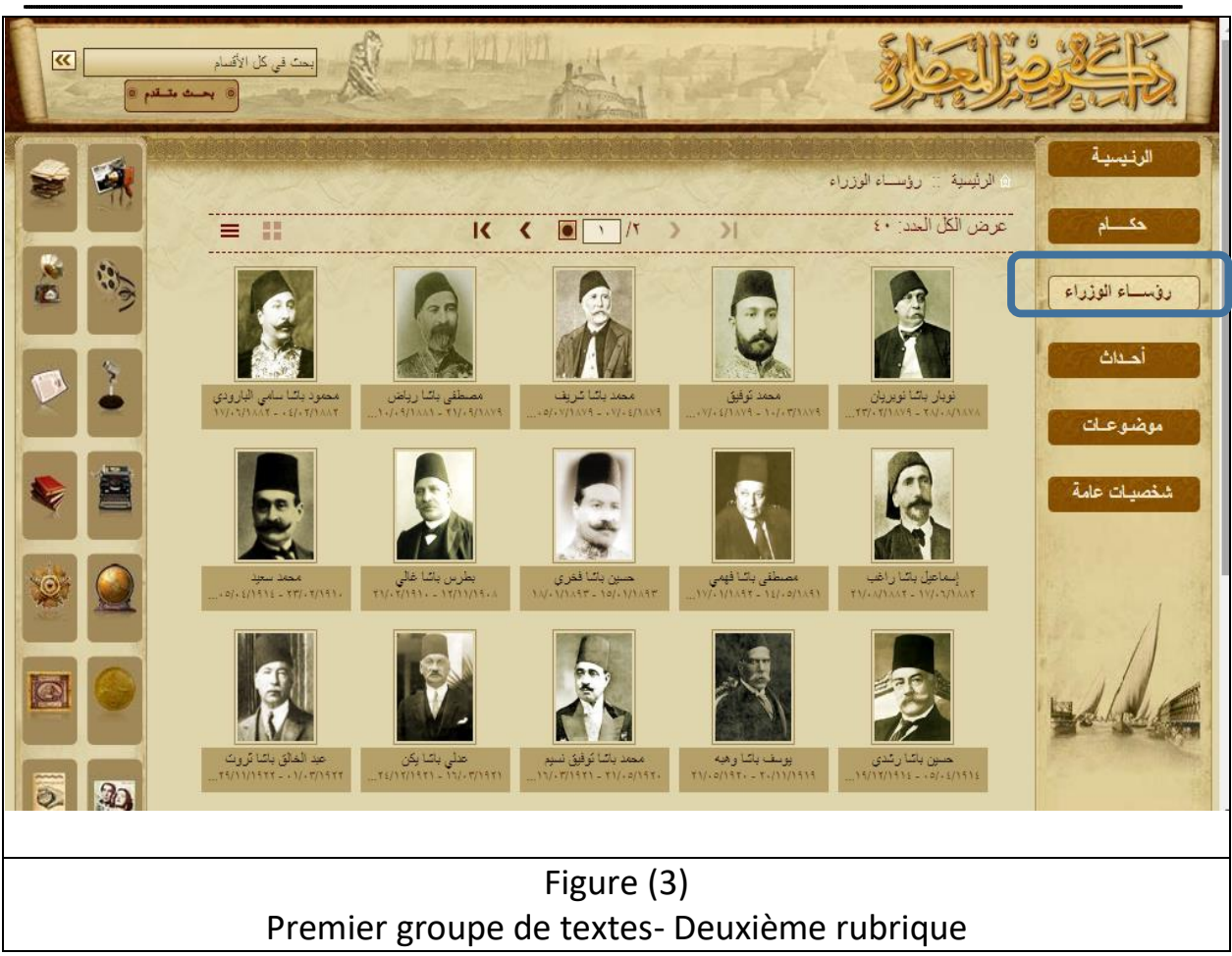

Le troisième groupe de textes (Figure 4$)^{7}$ fait partie d'une rubrique intitulée « événements » présentant un nombre d'événements historiques jalonnant l'histoire de l'Égypte moderne, comme:

- «L'expédition française en Egypte » en 1798,

- «L'accord du Condominium anglo-égyptien du Soudan » en 1899 ,

- «Le protectorat britannique sur l'Égypte » en 1914,

- «L'adoption de la constitution de 1923 » en 1923,

- «La visite du président Sadate à Jérusalem » en 1977.

${ }^{7}$ Cf., infra p.8 pour la figure (4) 


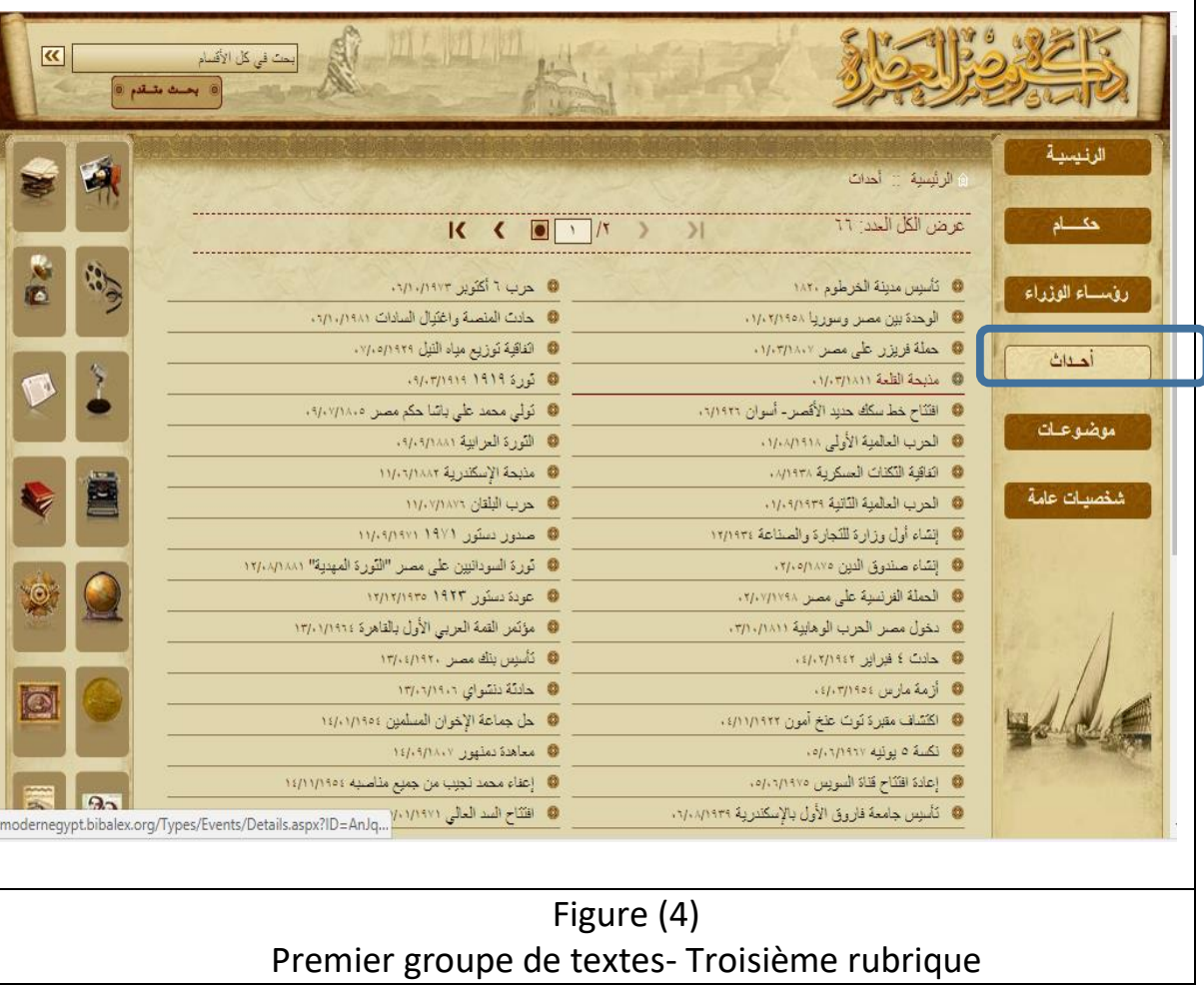

Le quatrième groupe de textes historiques (Figure 5$)^{8}$ fait partie d'une rubrique intitulée « sujets » et porte sur la vie scientifique, économique, sociale, politique et artistique à l'époque de Méhémet Ali. On y trouve des textes différents se rapportant à des thèmes comme:

- «L'éducation »,

- «Les milieux et les centres scientifiques et de recherche »,

- «Les voyages et les découvertes géographiques,

- «L'agriculture »...

- «Les constitutions égyptiennes »,

${ }^{8}$ Cf., infra p.9 pour la figure (5) 


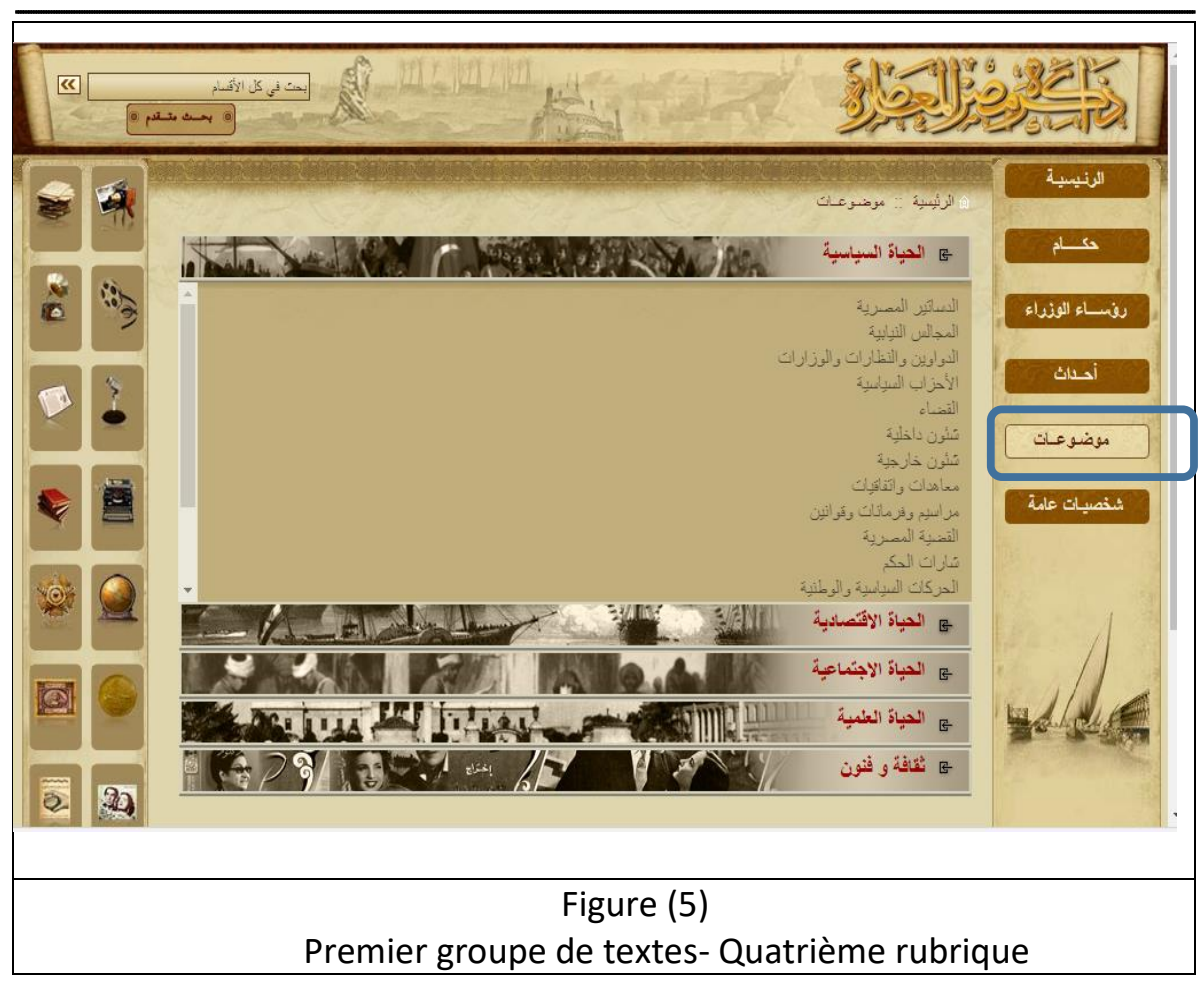

Le cinquième groupe de textes historiques (Figure 6) ${ }^{9}$ fait partie d'une rubrique intitulée «personnalités publiques». Ce sont des récits, résumant la vie d'un personnage célèbre qui a énormément influencé et enrichi la vie artistique et littéraire égyptienne dans la période allant de Méhémet Ali jusqu'à l'époque du président Sadat : un acteur, un metteur en scène, une chanteuse, un artiste, un homme politique, etc, comme:

- Omar Khayrat

- Ahmad Mazhar

- Adham Wanly

- Ismail Siri

${ }^{9}$ Cf., infra. p. 10 pour la figure (6) 


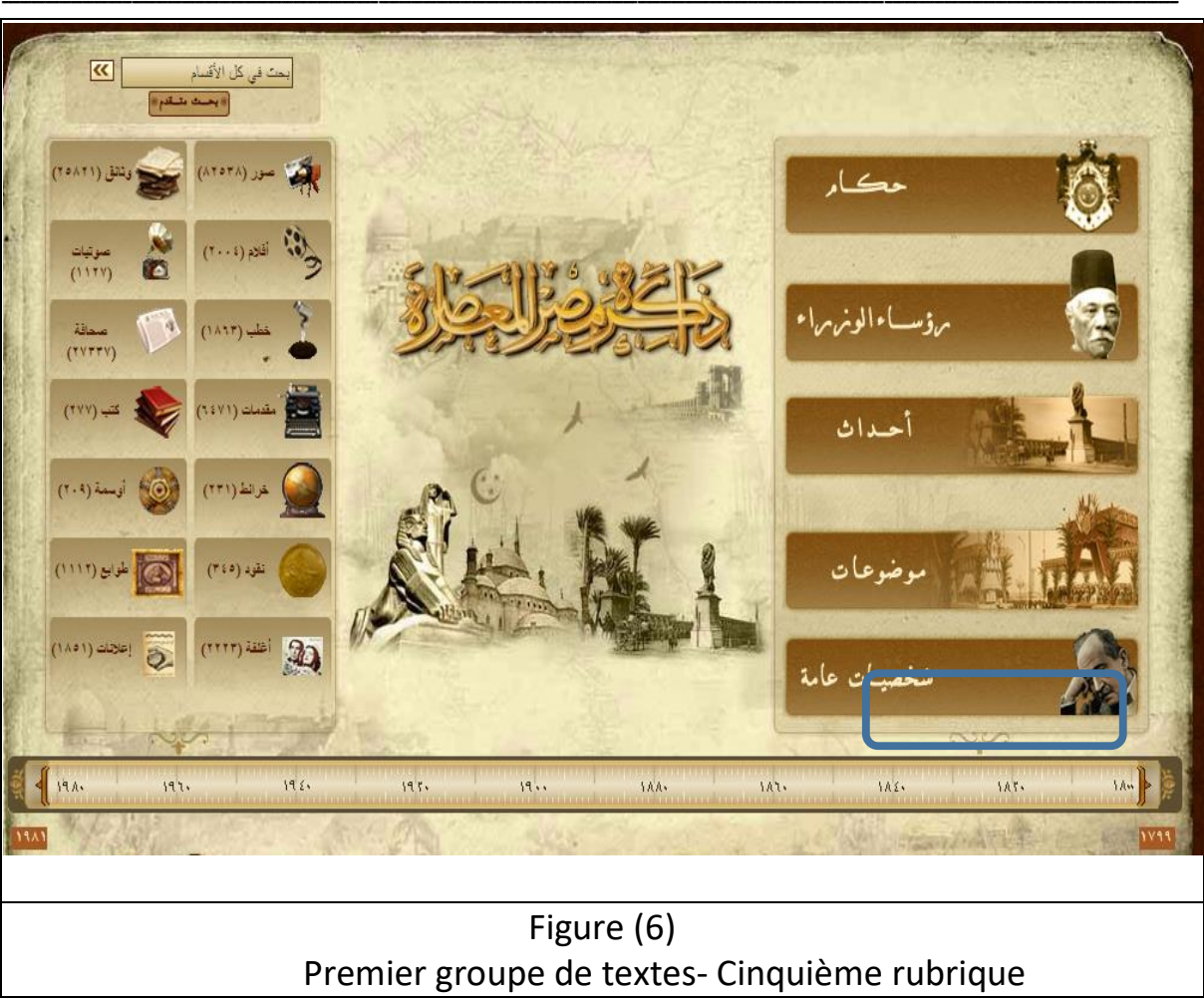

Le second volet, constitué de textes courts et intitulé «les archives» (Figure 7$)^{10}$, comprend différentes petites rubriques, à savoir: des légendes de photos, des documents, des titres de films, des enregistrements audios, des discours, des documents de presse, des recherches, des cartes, des photos de timbres, des livres, des photos de pièces de monnaies, des couvertures de magazines, des publicités, des médailles et des décorations.

${ }^{10}$ Cf. infra.p.11 pour la figure (7) 


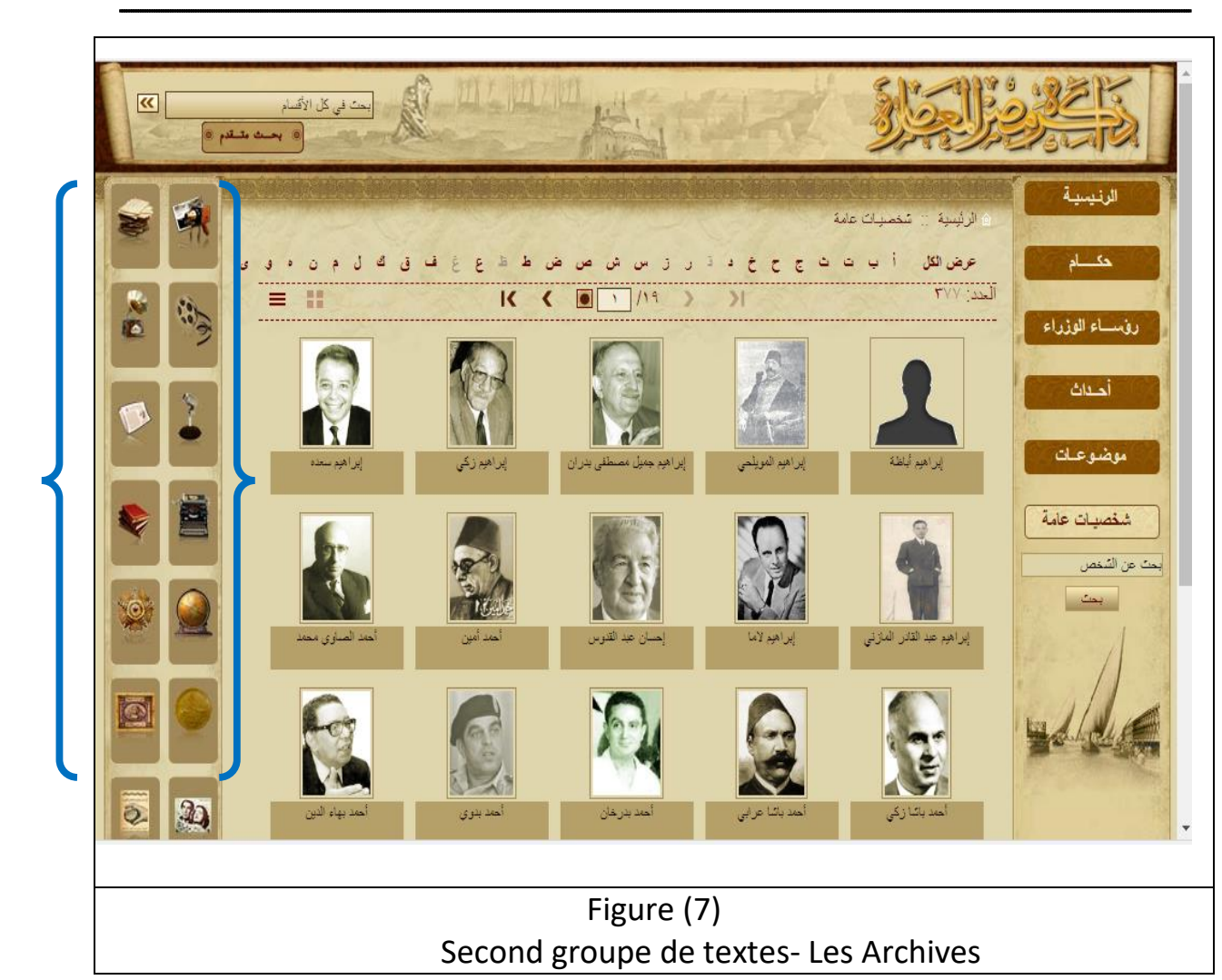

\section{Qu'est-ce qu'une erreur ou une impropriété en traduction?}

Avant de classer les erreurs et les impropriétés relevées tout le long du processus de traduction, il s'agit d'abord de définir ce que c'est qu'une erreur en général, d'évoquer ensuite les différents types d'erreurs ou d'impropriétés que peut commettre un traducteur et les raisons qui le conduisent à tomber dans de tels pièges traductionnels. En fait, la définition première de l'erreur est celle donnée par le Centre National de Ressources textuelles et lexicales (CNRTL): "Faute commise en se trompant $»$ ou encore «Chose fausse, erronée ${ }^{11}$. Une autre définition est celle proposée par le Dictionnaire de l'Académie

${ }^{11} \frac{\mathrm{http}: / / \mathrm{www} . c n r t l . f r / d e f i n i t i o n / \text { erreur }}{\text { Dernière consultation le 08/09/2019 }}$ 
française dans sa neuvième édition (Atlif) : «Action de se tromper, de s'écarter de la vérité » ou aussi : "Ce qui n'est pas conforme au vrai, au réel ou à une norme définie $»^{12}$. Une dernière définition est celle attestée par le dictionnaire français Larousse : «Acte de se tromper, d'adopter ou d'exposer une opinion non conforme à la vérité, de tenir pour vrai ce qui est faux » ainsi qu'une autre "Chose fausse, erronée par rapport à la vérité, à une norme, à une règle ${ }^{13}$.

$\mathrm{Si}$ nous avons défini dans les quelques lignes précédentes, la notion d'erreur en général, qu'en est -il alors pour la définition de l'erreur ou de l'impropriété en traduction ? En fait, la plupart des théoriciens et des enseignants de la traduction déclarent que toute faute en traduction est due à «l'incapacité du traducteur à faire passer, dans le texte d'arrivée, ce qu'il comprend et «savoure»du texte de départ » ${ }^{14}$. Ces erreurs, fautes ou impropriétés naissent, très prosaïquement, des situations où le traducteur ne comprend pas ou comprend mal le message qu'il doit traduire. La compréhension est, donc, au cœur du processus de traduction. Par conséquent, toute mécompréhension s'avère être la cause principale de toute erreur commise par un traducteur. Cette faute de compréhension peut être due à une série de facteurs. Tout d'abord, à un mauvais décodage linguistique : c'est-àdire à une mauvaise analyse morphologique, à une mauvaise analyse syntaxique (mauvais découpage), à une méconnaissance du lexique, ou enfin à une mauvaise analyse sémantique. Cette faute ou impropriété en traduction peut aussi avoir pour raison des erreurs dans les opérations cognitives : c'est-à-dire la construction de mauvaises inférences, l'absence de connaissances préalables permettant de reconstruire le non-dit (ellipses, sens vague, flou dans l'expression, métaphores), et la construction de présupposés erronés. ${ }^{15}$

${ }^{12} \mathrm{https}$ ://academie.atilf.fr/9/consulter/erreur?page $=1$ Dernière consultation le 08/09/ 2019

${ }^{13}$ Dictionnaire Larousse en ligne https://www.larousse.fr/dictionnaires/francais/erreur/30846?q=erreur\#30759 Dernière consultation le 08/09/ 2019

${ }^{14}$ Jeanne DANCETTE, « La faute de sens en traduction », in TTR (Traduction, terminologie, rédaction), Volume 2 , numéro 2, 2e semestre 1989, p.83.

${ }^{15}$ Cf. Ibid., p.93. 
En fait, rares sont les traducteurs qui n'ont jamais commis d'erreurs de traduction. Toutefois, certaines erreurs ont plus d'impact que d'autres. Ainsi, par exemple :

Vers 1830, Washington engage des discussions avec la France à propos d'une indemnité. Le ton est vif et le président des États-Unis, Andrew Jackson, propose des mesures exceptionnelles au Congrès. Le message que la France envoie à la Maison Blanche commence ainsi: «Le gouvernement français demande ...». Or, un secrétaire traduit cette phrase par "The French Government demands ...» ce qui ne signifie plus «demander» mais «exiger». La réaction du président américain est immédiate : il annonce que "si le gouvernement français ose exiger quoi que ce soit des États-Unis, il $n$ 'obtiendra rien ${ }^{16}$.

Heureusement, cette erreur fut corrigée et les discussions entre les deux gouvernements reprirent. La traduction donne lieu aussi parfois à des erreurs aux conséquences anodines ou amusantes. L'exemple cidessous en est la preuve:

«Après la crise économique de 2008, le groupe bancaire international HSBC s'est lancé dans une importante campagne publicitaire pour se donner un nouvel élan. Elle a imaginé un slogan simple, "Assume Nothing », que l'on peut traduire par "Ne supposez. rien » en français. Mais contre toute

\footnotetext{
${ }^{16}$ https://www.btb.termiumplus.gc.ca/tpv2guides/guides/chroniq/index-

fra.html?lang=fra\&lettr=indx_autr8KQ_bboMdGvY\&page=9X0H4FN_ZoEM.html \#note4

Dernière consultation le 06/02/ 2019
} 
attente, dans certains pays, la phrase choc s'est mystérieusement transformée en un « Do Nothing » qui n'avait rien de motivant pour les consommateurs.» ${ }^{17}$.

Cette erreur de traduction a coûté à la banque une perte de presque 10 millions de dollars.

Certaines fautes peuvent entraîner parfois des conséquences inattendues. Ainsi, dans les pays du golfe, la consommation de l'alcool, comme son utilisation en tant qu'ingrédient dans certains produits, est interdite. L'arrivée du chocolat «Milka Oreo » sur le marché a fait énormément de bruit car dans la recette du produit, figure l'expression «liqueur de chocolat». Aux Émirats arabes unis, cette information a causé un grand problème car les consommateurs ont cru que ce produit contenait de l'alcool. Les autorités ont retiré donc immédiatement ce produit du marché et l'ont soumis à des examens en laboratoires pour découvrir à leur grande surprise qu'aucune trace d'alcool n'a été trouvée. Cette erreur provient du fait que le terme «liqueur » avait été traduit comme une boisson alcoolisée sur la liste des ingrédients, alors que la liqueur de chocolat n'a rien à voir avec l'alcool ! ${ }^{18}$

Toutefois, le traducteur doit se montrer très prudent et très informé lorsqu'il traduit des textes d'importance capitale, comme ceux du Patrimoine. Il ne faut pas oublier que l'interprétation du traducteur joue un rôle fondamental dans sa traduction. Le traducteur doit donc être certain de traduire le sens exact du document et pour y parvenir, il doit s'informer des différences entre les langues et des différences de culture car s'il commet une erreur, toutes les techniques de traduction se révéleront impuissantes pour les corriger.

${ }^{17} \mathrm{Cf}$. https://beelingwa.com/fr/blog/erreur-de-traduction-10-exemples Dernière consultation le $06 / 022019$

${ }^{18}$ Cf. Ibid., Loc.cit 


\section{Les théories de traduction adoptées dans notre étude:}

La TiT ou La théorie du sens ou la théorie interprétative ${ }^{19}$ sert de théorie de base dans cette étude. La théorie interprétative repose sur un principe essentiel : la traduction n'est pas un travail sur la langue, sur les mots, c'est un travail sur le message. En d'autres termes, elle place le sens au centre de l'activité traduisante :

«On serait tenté de dire que les langues sont extérieures au processus de la traduction, elles sont le réceptacle du sens qui est exprimable dans n'importe quelle d'entre elles, elles ne se confondent pas avec lui ${ }^{20}$.

Cette théorie protège le traducteur de tomber dans le piège de la traduction littérale. En outre, le détachement de la forme originale du texte de départ assure une réexpression libre et claire des idées, ce qui facilite la compréhension par le public cible. De plus, cette théorie n'exige pas seulement la parfaite connaissance des langues source et cible, mais également une documentation assez fouillée afin de mieux maitriser le sujet du texte.

« Comprendre un texte c'est faire appel à une compétence linguistique et, simultanément, à un savoir encyclopédique.» ${ }^{21}$

${ }^{19}$ La théorie du sens ou la théorie interprétative de la traduction a été développé au sein de l'École Supérieure d'Interprètes et de traduction (ESIT, Paris). C'est pourquoi on l'appelle parfois " l'École de Paris". Elle a commencé à se développer à la fin des années soixante-dix (1970). On la doit essentiellement à Danica Seleskovitch et Marianne Lederer. La TIT est fondée sur l'observation de la pratique professionnelle de Danica Seleskovitch, qui s'est appuyée sur son expérience en tant qu'interprète de conférence pour formuler ce modèle de traduction.

${ }^{20}$ Dancia SELESKOVITCH, Marianne LEDERER: Interpréter pour traduire, Didier érudition, Paris, 1984, p.36.

21 Marianne LEDERER, La traduction aujourd'hui. Le modèle interprétatif, Hachette Paris, 1994, p.32. 
La théorie du Skopos a été également utilisée dans la traduction des aspects culturels des textes faisant l'objet de cette étude afin de répondre à la consigne du donneur d'ouvrage. Selon la consigne, le but de cette traduction est la transmission de l'histoire de l'Egypte à un public francophone tout en maintenant le plus grand nombre des culturèmes. Les textes cibles sont informatifs. Ainsi, la transmission correcte et complète du contenu du texte source, tout en respectant le génie de la langue cible, produira un texte qui remplit cette fonction. Les stratégies employées par le traducteur sont donc choisies en fonction de la finalité du texte d'arrivée. Il ne s'agit pas de la fonction assignée par l'auteur du texte original, mais la fonction assignée au texte cible par le commanditaire de la traduction dans la consigne de la traduction :

« C'est le client qui fixe au traducteur un but en fonction de ses besoins et de sa stratégie de communication ${ }^{22}$

\section{La typologie des problèmes de traduction dans le corpus étudié :}

Le traducteur affronte plusieurs problèmes lors de la traduction d'un texte. Ces problèmes peuvent être classés en plusieurs types : problèmes culturels, problèmes lexico-sémantiques, problèmes syntaxiques, problèmes techniques, etc. Pour résoudre ces différents problèmes, le traducteur recourt à des stratégies diverses de traduction choisies en fonction du skopos de la traduction et de la fonction du texte dans la langue cible. Nous avons eu recours aux procédés proposés par Vinay et Darbelnet dans leur Stylistique comparée du français et de l'anglais $\mathbf{2 3}^{\mathbf{3}}$.

En révisant et corrigeant la traduction des textes faisant l'objet du corpus étudié, nous avons pu relever un certain nombre de problèmes qui auraient pu être considérés comme des erreurs de traduction flagrantes ou des impropriétés de traduction si elles n'avaient pas été

\footnotetext{
22 Mathieu GUIDÈRE, Introduction à la traductologie, De Boeck, Bruxelles, 2008, p.73.

${ }^{23}$ Cf .J.-P.VINAY : Stylistique comparée du Français et de l'Anglais, Didier, 2004.
} 
révisées et corrigées pour paraitre dans leur version finale telles qu'elles seront présentées dans cette étude.

Ces problèmes peuvent être classés comme suit :

\subsection{Problèmes lexico-sémantiques:}

«Les problèmes lexicaux sont des problèmes qui se posent devant le traducteur parce qu'il n'arrive pas à saisir le sens d'un mot ou d'une expression ou il ne trouve pas un équivalent dans la langue cible. La polysémie, les collocations, les expressions idiomatiques, les noms propres sont les problèmes lexicaux les plus communs ». ${ }^{24}$

\subsubsection{Noms propres :}

Les noms propres constituent un problème important dans la traduction, surtout dans le cas où les deux langues envisagées utilisent des systèmes graphiques différents. Ainsi, par définition, le nom propre est :

«une sous-catégorie des noms qui sémantiquement, se réfèrent à un objet extralinguistique, spécifique et unique, distingué par sa dénomination des objets de même espèce» ${ }^{25}$.

Les étudiants avaient affronté quelques difficultés dans la classification de ces noms propres tout comme dans leur traduction. Ces difficultés provenaient d'abord du fait qu'ils n'avaient adopté aucune classification des noms propres ou qu'ils ne savaient pas parfois comment les traduire exactement: devraient-ils recourir au calque $?^{26}$ à l'emprunt $?^{27}$ à la translittération ${ }^{28}$ ? ou à d'autres procédés?

${ }^{24}$ Hasan GHAZALA, Translation as Problems and Solutions: course-book for university students and trainee translators. Dar Al-alam, Beyrouth, 1995, p.24.

${ }^{25} \mathrm{G}$. DUBOIS, Dictionnaire de linguistique, Paris, Larousse, 1973.

${ }^{26}$ Le calque en traduction est un procédé qui consiste à traduire littéralement un mot ou une expression : on peut parler de mot à mot. Parfois, cela fonctionne très bien et le sens est correctement retranscrit. Au contraire, il arrive que calquer une expression, une phrase ou un mot ne permet pas de traduire fidèlement l'idée. https://sites-formations.univ-rennes2.fr/lea-cfttr/veille/2016/06/24/le-calque-entraduction/

Dernière consultation le 09/02/2021 
Nous avons alors expliqué aux étudiants que la classification réalisée par le linguiste germanophone Bauer ${ }^{29}$ est la plus adoptée dans la catégorisation des noms propres( Figure 8$)^{30}$. Dans cette classification, Bauer énumère ce qui, par convention, constitue un nom propre et prend en considération des éléments extra-linguistiques propres au référent. Cette typologie comporte 5 classes ou cinq hypertypes : Anthroponymes (noms de personnes individuelles et groupes) ; Toponymes (noms de lieux) ; Ergonymes (objets et produits manufacturés) ; Praxonymes (faits historiques, maladies, événements culturels); Phénonymes (ouragans, zones de pressions, astres et comètes) ${ }^{31}$. Nous avons essayé également de discuter avec eux des problèmes des procédés de traduction appliqués généralement aux noms propres pour aboutir à la traduction la plus adéquate et conserver le plus grand nombre de culturèmes.

Un calque peut également porter sur certaines conventions de la langue ou certaines de ses particularités. Le calque consiste à former des mots ou des expressions en combinant des formes indigènes sur un modèle étranger.

https://www.researchgate.net/publication/336920242_Calque_linguistique_et_transf ert_semantique

Dernière consultation le 09/02/2021

${ }^{27} \mathrm{~L}$ 'emprunt consiste à intégrer une unité lexicale transférée à partir d'une autre langue telle quelle, avec son signifiant et son signifié moyennant une certaine adaptation au système morphologique et phonétique de la langue d'arrivée quand cela s'impose.

https://www.researchgate.net/publication/336920242_Calque linguistique et transf ert_semantique

Dernière consultation le 09/02/2021

${ }^{28} \mathrm{La}$ translittération est la transcription signe par signe d'un système d'écriture en un autre système.

https://www.cnrtl.fr/definition/translitt\%C3\%A9ration

Dernière consultation le 09/02/2021

${ }^{29}$ G. BAUER: Namenkunde des Deuschen, Germanistische Lehrbuchsammlung Band, (1985),p. 21.cité par Béatrice DAILLE, Nordine FOUROUR \& Emmanuel MORIN : "Catégorisation des noms propres : une étude en corpus », in Cahiers de Grammaire 25 (2000), « Sémantique et Corpus », p.122.

${ }^{30} \mathrm{Cf}$., infra p. 18 pour la Typologie des noms propres selon Bauer

${ }^{31} \mathrm{Cf}$. Béatrice DAILLE, Nordine FOUROUR \& Emmanuel MORIN :

«Catégorisation des noms propres : une étude en corpus », in Cahiers de

Grammaire 25 (2000), « Sémantique et Corpus », pp. 115-129. 


\begin{tabular}{|c|c|}
\hline \multicolumn{2}{|l|}{ ANTHROPONYMES } \\
\hline Patronymes & Dupont, Durant \\
\hline Prénoms & Alexandre, Jean-Paul \\
\hline Ethnonymes & L'Italien, les Français \\
\hline${ }^{*}$ Partis et autres organisations & Le PCF, l'ONU, I'Union européenne \\
\hline $\begin{array}{l}\text { "Ensembles artistiques, groupes } \\
\text { musicaux et troupes de théâtre }\end{array}$ & $\begin{array}{l}\text { Les Toten Hosen, l'orchestre } \\
\text { philharmonique de New York }\end{array}$ \\
\hline Pseudonymes & L'Ange vert \\
\hline Zoonymes & Médor \\
\hline \multicolumn{2}{|l|}{ TOPONYMES } \\
\hline Toponymes > Pays & Europe \\
\hline Pays & France, Sahara occidental \\
\hline${ }^{*}$ Pays $<$ Toponymes $>$ Villes & l'le de France \\
\hline Villes & Paris, Belo Horizonte \\
\hline Microtoponymes & Le Quartier Latin, Prenzlauerberg \\
\hline Hydronymes & La Manche, la Seine, le lac Ontario \\
\hline Oronymes & Les Andes, les Alpes \\
\hline Rues & $\begin{array}{l}\text { La rue de la Paix, le Faubourg Saint } \\
\text { Honoré }\end{array}$ \\
\hline Déserts & Le Sahara, le désert de Gobi \\
\hline Édifices & $\begin{array}{l}\text { La Maison Blanche, la gare } \\
\text { Montparnasse }\end{array}$ \\
\hline \multicolumn{2}{|l|}{ ERGONYMES } \\
\hline Sites de production & Renault Wilword \\
\hline Marques de produits & Coca, Kleenex, Scotch \\
\hline Entreprises industrielles & $\begin{array}{l}\text { Microsoft Corporation, Sud-Marine } \\
\text { industrie }\end{array}$ \\
\hline Coopératives & Semences de Provence \\
\hline $\begin{array}{l}\text { Établissement d'enseignement et de } \\
\text { recherche }\end{array}$ & Université de Nantes \\
\hline Installations militaires & la ligne Maginot \\
\hline "Euvres intellectuelles & Matrix, l'Écume des jours \\
\hline \multicolumn{2}{|l|}{ PRAXONYMES } \\
\hline Faits historiques & La Guerre de Cent Ans \\
\hline Maladies & La maladie d'Alzheimer \\
\hline $\begin{array}{l}\text { "Événements culturels, sportifs, } \\
\text { commerciaux, etc. }\end{array}$ & Le Festival du film de Berlin \\
\hline "Période historique & le Paléolitique \\
\hline \multicolumn{2}{|l|}{ PHENONYMES } \\
\hline Catastrophes naturelles & Le cyclone Mitch \\
\hline Astres et comètes & La comète de Halley \\
\hline \multicolumn{2}{|c|}{ Figure (8) } \\
\hline \multicolumn{2}{|c|}{ Typologie des noms propres selon Bauer ${ }^{32}$} \\
\hline
\end{tabular}

${ }^{32}$ G. BAUER: Namenkunde des Deuschen, Germanistische Lehrbuchsammlung Band , (1985),p. 21.cité par Béatrice DAILLE, Nordine FOUROUR \& Emmanuel MORIN, Op.cit, p.122. 


\subsubsection{Ergonymes:}

Les Ergonymes couvrent, dans le corpus étudié, les titres des films, des œuvres littéraires, des œuvres musicales. Ils comprennent également les titres des journaux et des revues. Dans la traduction de cette rubrique, les étudiants que nous supervisions avaient adopté plusieurs procédés de traduction : il y avait parmi eux, ceux qui ont uniquement translitéré le nom, d'autres avaient calqué ou traduit littéralement en langue cible, le nom arabe. Or, puisqu'il s'agit de noms appartenant au Patrimoine culturel égyptien, il faut essayer tant que possible de diminuer l'écart culturel entre le texte de départ et le texte d'arrivée. Autrement dit, pour faire "revivre un texte dans un contexte culturel cible $»^{33}$, le traducteur devra opter pour une démarche particulière qui vise à assurer le transfert interlingual de la marque culturelle de la culture source dans la culture réceptrice.

\section{- Exemple (1): مجلت روضت المدارس}

\begin{tabular}{|c|c|c|}
\hline Notre traduction & $\begin{array}{l}\text { Traduction proposée par } \\
\text { les étudiants }\end{array}$ & Texte source \\
\hline $\begin{array}{l}\text { En } 1870 \text {, il fut engagé } \\
\text { comme rédacteur en } \\
\text { chef de la revue Rawdat } \\
\text { al-madâris (Le Jardin } \\
\text { d'enfants des écoles } \\
\text { égyptiennes) } \\
\text { par Ali Moubarek. fondée }\end{array}$ & $\begin{array}{l}\text { En } 1870, \text { il fut nommé } \\
\text { rédacteur en chef de la } \\
\text { revue Jardin des écoles, } \\
\text { créée par Ali Moubarek. }\end{array}$ & 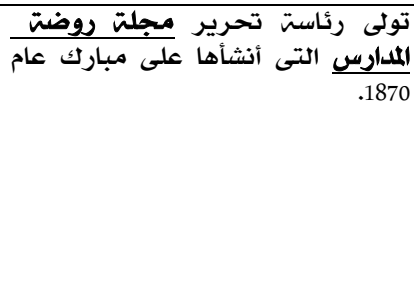 \\
\hline
\end{tabular}

33 Georgiana I. BADEA : «La traduction de l'écart culturel», in https://www.academia.edu/1249770/La traduction_de_lecart_culturel

Dernière consultation le 18/07/2020

${ }^{34}$ Pour plus d'informations sur la revue Rawdat al-madâris (Le Jardin d'enfants des écoles égyptiennes), veuillez consulter l'article de Bertrand MILLET : «Genèse de la presse enfantine en Egypte 1870-1915», pp.25-35 in https://books.openedition.org/cedej/512?lang=fr\#text

Dernière consultation le 19/07/2020 
- Exemple (2):الشريد

\begin{tabular}{|c|c|c|}
\hline Notre traduction & $\begin{array}{c}\text { Traduction proposée par } \\
\text { les étudiants }\end{array}$ & Texte source \\
\hline $\begin{array}{l}\text { En 1942, il réalisa son } \\
\text { premier film intitulé Al- } \\
\text { chârid }^{35} \text { (Le vagabond). }\end{array}$ & $\begin{array}{l}\text { En 1942, il réalisa son } \\
\text { premier film intitulé " Le } \\
\text { vagabond ». }\end{array}$ & فيلم من أخراجه وهو ألشراله عام 1942 فى \\
\hline
\end{tabular}

Dans les exemples ( 1 ) et ( 2 ), nous avons adopté presque les mêmes procédés pour traduire le nom de la revue et le nom du film : la translittération et la traduction littérale. Ainsi, pour les deux exemples, les titres ont été translitérés, et nous avons ajouté une traduction littérale entre parenthèses. Cette stratégie nous a permis à la fois de maintenir l'appellation originale et d'expliquer au lecteur cible ce qui se cache derrière cette appellation. Cependant, pour le titre de la revue، nous avons fait un ajout du syntagme prépositionnel 《d'enfants 》 pour donner à 《روضة 》 son sens correct en arabe qui signifie l'école maternelle:

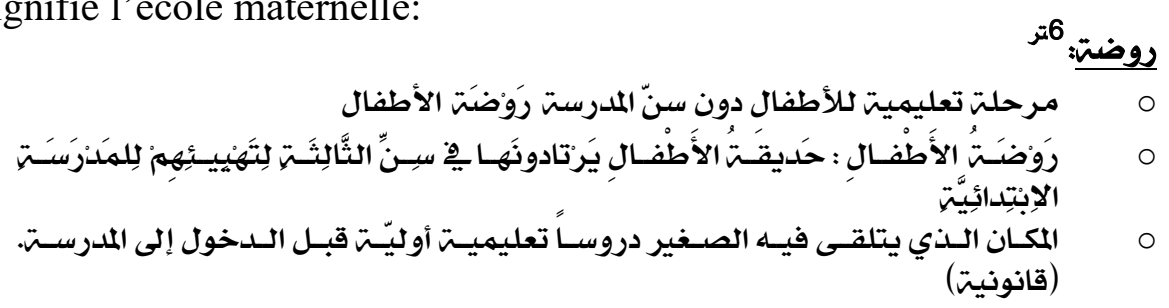

Nous avons, en plus, ajouter l'adjectif « égyptiennes » à écoles pour restituer le titre correct de la revue en arabe qui n'est pas présent dans la version source (Figure 9) ${ }^{37}$. Nous avons ainsi abouti à la traduction suivante «Le Jardin d'enfants des écoles égyptiennes» qui correspond au titre original de la revue «روضت المدارس المصريت"

${ }^{35}$ Pour plus d'informations sur le film Al-chârid (Le vagabond), veuillez consulter le site :

https://www.marefa.org/\%D8\%A7\%D9\%84\%D8\%B4\%D8\%B1\%D9\%8A\%D8\%A F (\%D9\%81\%D9\%8A\%D9\%84\%D9\%85)

Dernière consultation le 19/07/2020

${ }^{36} \mathrm{https} / / /$ www.almaany.com/ar/dict/ar-ar/\%D8\%B1\%D9\%88\%D8\%B6\%D8\%A9/ Dernière consultation le 19/07/2020

${ }^{37}$ Cf., infra p.21 pour la version originale de la première page de la revue « Le Jardin d'enfants des écoles égyptiennes» ou 《) 


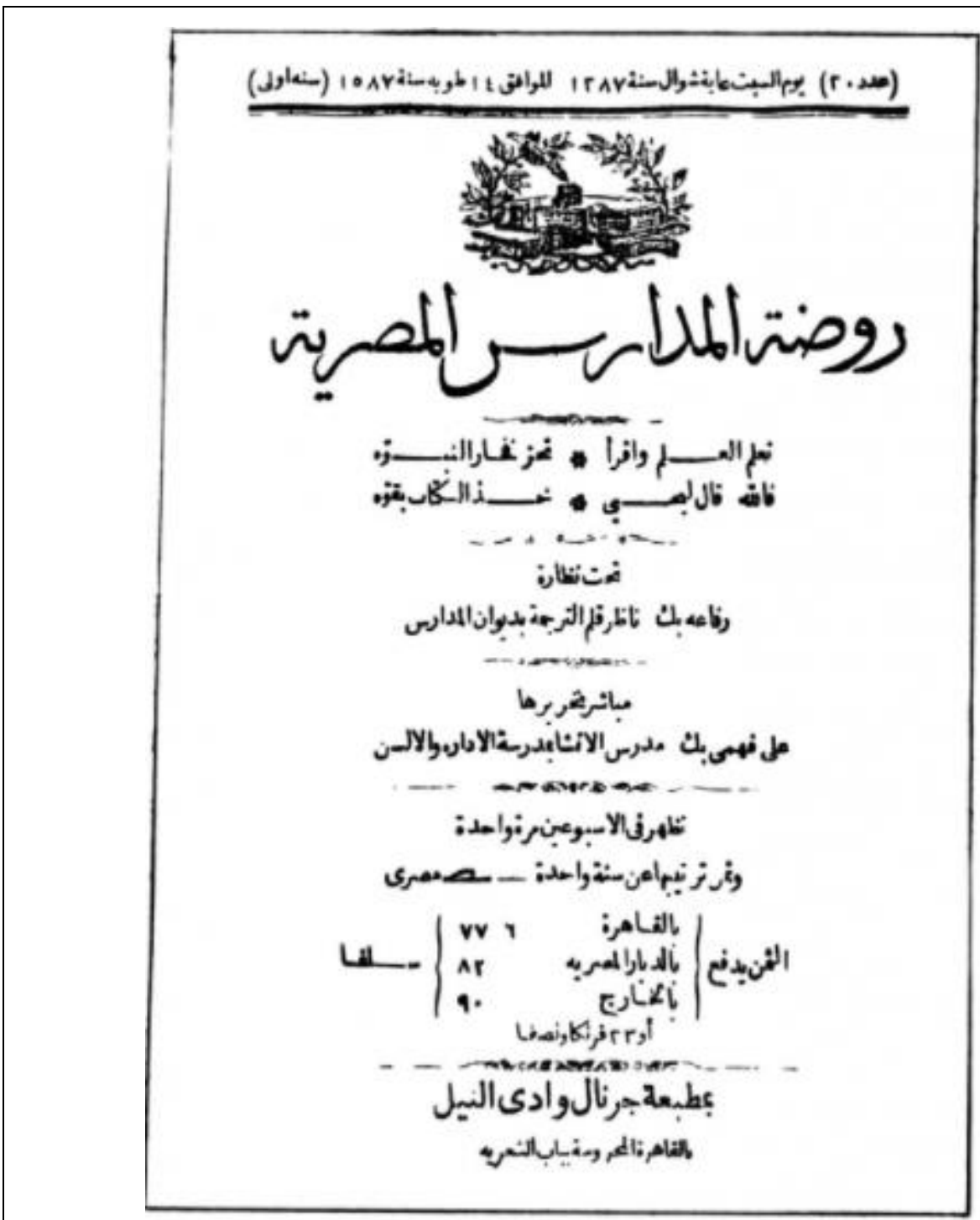

Figure (9)

La première page de la revue "Le Jardin d'enfants des écoles égyptiennes» 
- Exemple: (3) السكرية

\begin{tabular}{|c|c|c|}
\hline Notre traduction & $\begin{array}{c}\text { Traduction proposée par } \\
\text { les étudiants }\end{array}$ & Texte source \\
\hline $\begin{array}{l}\text { Al-sukaria }^{38} \text { (Le Sucrier, } \\
\text { publié en français sous le } \\
\text { titre Le Jardin du passé). }\end{array}$ & Le sucrier & السكريتي \\
\hline
\end{tabular}

Pour la traduction du titre du roman de Naguib Mahfouz "السكرية", qui fait partie d'une trilogie ${ }^{39}$ très célèbre adaptée au cinéma, et qui fait également référence à un quartier du vieux Caire qui porte le même nom, tout comme les deux autres romans faisant partie de cette même trilogie, certains étudiants avaient traduit le titre du roman en «Le Sucrier », d'autres l'avaient translitéré uniquement. Cependant, ces deux solutions ne se sont pas avérées suffisantes pour assurer le transfert du sens de la langue source à la langue cible. Ainsi, pour résoudre ce problème, et comme la plupart des romans de Naguib Mahfouz ont été traduits en français par Philippe Vigreux et publiés en France, nous avons adopté plusieurs procédés : la translitération du

${ }^{38}$ Le roman de Naguib Mahfouz « Al-sukaria » a été traduit en français par Philippe Vigreux et publié en 1991.

${ }^{39}$ La trilogie de Naguib Mahfouz :Impasse des deux palais, Le jardin du passé et La palais du désir.
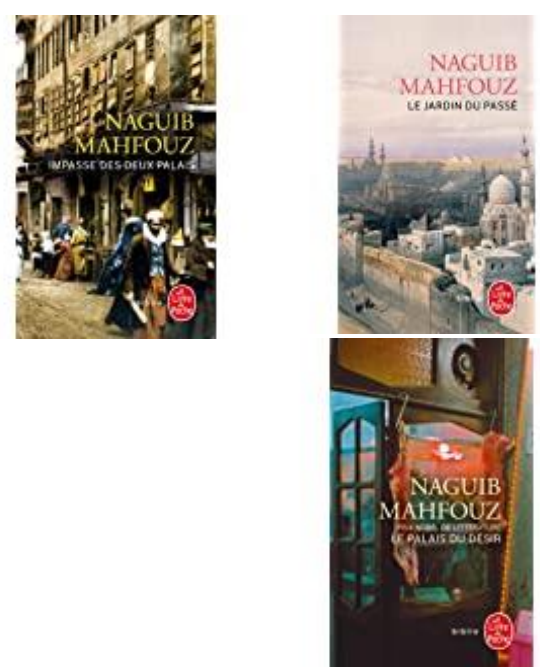
titre arabe d'abord «Al-sukaria »; ensuite, la traduction littérale du titre arabe entre parenthèses «Le Sucrier »; et enfin, l'étoffement en ajoutant le titre sous lequel le roman a été publié en français et qui fonctionne déjà dans la culture cible « Le Jardin du passé ».

\subsubsection{Toponymes:}

«Les toponymes constituent un sous-ensemble de la catégorie des noms propres. On considère habituellement qu'ils ne suivent pas la norme de la langue courante et qu'ils peuvent prendre des formes diverses sans suivre nécessairement les règles grammaticales de la langue et sans avoir nécessairement un contenu sémantique ${ }^{40}$ Il s'agit d'un nom propre qui désigne un lieu : nom de villes et de pays, noms de rues et de quartiers, des géonymes, noms de mers et d'hydronymes, nom de ponts et de jardins, etc...Dans le corpus étudié, les toponymes couvraient des noms de villes et de capitales, des noms de rues et de quartiers, des noms de constructions.

\subsection{Noms des villes et capitales :}

La traduction des noms des pays et des villes pose également un problème devant tout traducteur surtout si le nom n'a pas un équivalent normalisé par l'ONU dans la langue cible. Importer un nom de lieu dans une langue autre que celle parlée dans la zone linguistique où est situé le lieu géographique implique très souvent une adaptation du toponyme aux règles de la langue cible sur tous les niveaux linguistiques (orthographique, phonologique, morphologique, syntaxique, pragmatique). Le résultat de cette adaptation est une forme spécifique de toponyme, appelée l'exonyme. D'après la définition de l'ONU, un exonyme est un nom géographique utilisé dans une langue pour désigner un lieu situé en dehors du territoire dont cette langue est la langue officielle. Or, ce même toponyme tel qu'il est prononcé et écrit dans la langue parlée dans la zone géographique dans laquelle se trouve l'objet topographique est appelé

\footnotetext{
${ }^{40}$ Jonas LÖFSTRÖM, Betina SCHNABEL : «Comment analyser et comparer les toponymes de différentes langues dans une perspective synchronique », in Nouvelle revue d'onomastique [en ligne], $\mathrm{n}^{\circ} 52$, 2010. pp. 291-318

https://www.persee.fr/doc/onoma_0755-7752_2010_num_52_1_1549

Dernière consultation le 09/09/2020
} 
$\overline{\text { endonyme }^{41} \text {. L'ONU le définit comme appellation toponymique }}$ exprimée dans la forme orthographique et dans la langue locale de la région où l'entité dénommée est située, indépendamment du système d'écriture. ${ }^{42}$. Nous avons donc utilisé ces exonymes historiques déjà connus dans la culture cible.

- Exemples (4):

\begin{tabular}{|c|c|c|}
\hline Notre traduction & $\begin{array}{c}\text { Traduction proposée par } \\
\text { les étudiants }\end{array}$ & Texte source \\
\hline L'Égypte. & L'Egypte & مصر \\
\hline Le Levant & Le Moyen Orient/Sham & الشام \\
\hline Damas & Dimašq & دمشق \\
\hline $\begin{array}{l}\text { Médine (Al-Madina } \\
\text { Munawwara, deuxième ville } \\
\text { sainte de I'Islam après la } \\
\text { Mecque) }\end{array}$ & $\begin{array}{l}\text { Al-Madīna/ Al-Madīna al- } \\
\text { Munawwara }\end{array}$ & المدينتة \\
\hline Kaboul. & Kabel & كابل \\
\hline
\end{tabular}

Pour la traduction de ces toponymes, les étudiants n'avaient pas suivi les règles de traduction propres aux toponymes. Autrement dit, ils ont translitéré certains noms bien que ces endonymes aient des exonymes français attestés par les experts de l'exonymisation des toponymes de l'ONU ; ils ont transcrit certains autres selon leur prononciation dans la langue de départ, ce qui n'est pas correct sauf si ces endonymes n'ont pas d'exonymes connus et approuvés. Nous leur avons donc expliqué que pour la traduction des toponymes, il faut d'abord voir le nom adopté et attesté dans la langue cible, ensuite si ce nom n'a pas d'équivalent, on peut le translitérer en utilisant le système phonologique propre à chaque langue et en faisant les adaptations nécessaires au niveau orthographique ${ }^{43}$.

Ainsi, «Egypte, Damas, Kaboul, Le Levant, Médine» sont des endonymes qui ont des exonymes dans la langue source. Cependant,

\footnotetext{
${ }^{41}$ Marc RICHARD: « Des dénominations multiples de lieux dans la perspective de la normalisation des noms géographiques» Working Paper in Commission de toponymie du Québec, n.17, 16 mai 1989, p.4

${ }^{42}$ Cf., Jonas LÖFSTRÖM, Betina SCHNABEL, Op.cit. p.300.

https://www.persee.fr/doc/onoma_0755-7752_2010_num_52_1_1549

Dernière consultation le 09/09/2020

${ }^{43}$ Cf., Jonas LÖFSTRÖM, Betina SCHNABEL, Op.cit. p.302.

https://www.persee.fr/doc/onoma_0755-7752_2010_num_52_1_1549
} 
pour l'exonyme «Médine », nous avons eu recours à deux procédés : la translitération et l'étoffement. Nous avons translitéré le nom de la ville «Al-Madīna al-Munawwara » comme il est prononcé dans la langue source et nous lui avons ajouté une explication entre parenthèses «deuxième ville sainte de l'Islam après la Mecque » car il s'agissait ici d'une ville sainte, qui diffère de toutes les villes arabes dont les caractéristiques pourraient être méconnues pour tout lecteur étranger.

\subsection{Noms des quartiers et des rues:}

- Exemple ( 5 ):

\begin{tabular}{|c|c|c|}
\hline Notre traduction & $\begin{array}{c}\text { Traduction proposée par } \\
\text { les étudiants }\end{array}$ & Texte source \\
\hline $\begin{array}{l}\text { Le quartier d'al-Fagãlah } \\
\text { (Quartier populaire dont } \\
\text { le nom provient de } \\
\text { « vendeurs de radis » qui } \\
\text { résidaient jadis dans cette } \\
\text { région) }\end{array}$ & Le quartier d'al-Fagãlah & حى الفجالة \\
\hline
\end{tabular}

Pour traduire le nom du quartier, les étudiants avaient uniquement translitéré le nom arabe «al-Fagãlah » afin de maintenir la couleur locale et parce qu'il n'y avait pas un équivalent français. Or, ce toponyme ne signifie rien pour un lecteur occidental, nous avons donc suggéré aux étudiants d'avoir recours à «l'incrémentialisation qui est une traduction explicative destinée à éclairer le lecteur ignorant de la réalité culturelle de la région de la langue cible ${ }^{44}$. Nous avons donc ajouté une explication entre parenthèses pour montrer l'origine du nom (Quartier populaire dont le nom provient de « vendeurs de radis » qui résidaient jadis dans cette région). Ainsi, nous avons tenté de préserver le plus grand nombre de culturèmes tout en élucidant la partie qui pourrait être non- compréhensible.

${ }^{44}$ Claire AGAFONOV, Thierry GRASS et al. : « La traduction multilingue des noms propres dans PROLEX », in M3TA, Volume 51, numéro 4, décembre 2006, p.327. 
5.1.1.2.3. Noms des constructions (pont, barrage) :

- Exemple: ( القناطر الخيريت)

\begin{tabular}{|c|c|c|}
\hline Notre traduction & $\begin{array}{c}\text { Traduction proposée par } \\
\text { les étudiants }\end{array}$ & Texte source \\
\hline $\begin{array}{c}\text { Al-Qanater Al-Khaïriya } \\
\text { (Le Barrage du Delta) }\end{array}$ & $\begin{array}{c}\text { Al-Qanater Al-Khaïriya } \\
\text { (Barrages de la prospérité) }\end{array}$ & \\
\hline
\end{tabular}

Dans la traduction des noms de constructions, il faut adopter la même stratégie que dans la traduction de tout nom propre: soit trouver l'exonyme correspondant dans la langue cible, soit le translitérer uniquement, soit enfin, le translitérer et ajouter une explication entre parenthèses s'il y a un élément à élucider. Dans la traduction du nom du Barrage, les étudiants avaient utilisé deux procédés de traduction : la translittération et le calque afin de maintenir l'appellation originale du barrage tout en expliquant au lecteur ce qui se cache derrière cette appellation. En fait, en calquant le nom arabe du barrage, ils voulaient expliquer au lecteur étranger le rôle du barrage à cette époque : il était considéré comme une source de prospérité parce qu'il irrigue les terrains du Delta.

Or, ces deux procédés auraient été corrects s'il n'y avait pas un équivalent français au nom de ce barrage. Nous avons donc maintenu le premier procédé de translitération du nom du barrage «Al-Qanater Al-Khaïriya » et nous avons ajouté entre parenthèses l'exonyme français du nom du barrage «Le Barrage du Delta» que nous avons trouvé dans différents contextes ${ }^{46}$. Donc, nous avons utilisé comme procédés : la translitération et l'équivalence.

\subsubsection{Anthroponymes: (Noms de personnes)}

Les noms des personnes ou anthroponymes, sont une réalité linguistique puisqu'ils sont des messages verbaux dont les signes sont ceux de la langue quotidienne. Ils sont aussi une réalité ethnologique car ils sont le lieu de l'expression culturelle, dans sa genèse et son contenu. Ils sont également une réalité psychosociale car ils affirment

\footnotetext{
${ }^{45}$ https://hebdo.ahram.org.eg/NewsContent/1011/32/97/5046/AlQanater,-unmod\%C3\%A8le-dirrigation-moderne.aspx Dernière consultation le 09/11/2020

${ }^{46}$ https://www.sis.gov.eg/Story/84113/Al-Qanater-Al-Kha\%C3\%AFriya?lang=fr Dernière consultation le 09/11/2020
} 
et renforcent le réseau des relations dans lequel l'individu se définit socialement et dans lequel s'épanouit sa personnalité. Ils sont enfin, une réalité politique car ils sont l'objet de décisions au sein d'un conflit entre la tradition et la modernité. L'anthroponymie n'est donc pas uniquement un inventaire de noms, mais le témoin et le reflet d'une culture, d'une attitude, parfois même d'un engagement à l'égard de la société ${ }^{47}$.

Dans le corpus en question, les anthroponymes étaient en grand nombre : des anthroponymes de souverains, de présidents, d'écrivains, de chanteurs, de militants, etc. Nous avons choisi quelques exemples des noms propres qui ont constitué des pièges traductionnels pour les étudiants.

- Exemples: ( 7 )

\begin{tabular}{|c|c|c|}
\hline Notre traduction & $\begin{array}{c}\text { Traduction proposée par } \\
\text { les étudiants }\end{array}$ & Texte source \\
\hline Le Khédive Abbas Hilmi & Le Khédive Abbās Ḥilmī & الخديوي عباس حلمي \\
\hline $\begin{array}{l}\text { Le Président Anouar El- } \\
\text { Sadate }\end{array}$ & $\begin{array}{l}\text { Le Président Anwar as- } \\
\text { Sādāt /Anouar al Sadate }\end{array}$ & الرئيس أنور السادات \\
\hline Le Roi Fouad I $^{\mathrm{er}}$ & Le Roi Fouad le Premier & الملكك فؤاد الأول \\
\hline Méhémet-Ali Pacha & Muḥammad Ali Pacha & محمد علي باشا \\
\hline
\end{tabular}

Certains étudiants ont eu recours, dans la traduction de ces noms propres, au procédé de la translitération du nom en français pour qu'il ait la même prononciation arabe, à l'exemple de أنور السادات الرئيس . Ces noms propres ont été traduits par les étudiants en «Le Président Anwar as-Sādāt », et «Le Khédive Abbās Ḥilmī » ce qui n'est pas faux puisque nous sommes avant tout des arabes et nous avons le droit de maintenir la même prononciation du nom que celle présente dans la langue de départ. D'autres étudiants avaient choisi de transcrire le nom selon son équivalent dans la langue cible à

\footnotetext{
${ }^{47}$ Cf., Paul Edwin MALEKOU : Les anthroponymes et toponymes Gisir: proposition d'un modèle de dictionnaire, Maîtrise 2006, Université Omar BONGO .

https://www.memoireonline.com/07/08/1412/m_anthroponymes-toponymes-gisirproposition-modele-dictionnaire $5 . \mathrm{html}$

Dernière consultation le 03/08/2020
} 
l'exemple de «Anouar al-Sadate», ce qui est également correct comme le montrent les différents contextes consultés ${ }^{48}$.

Par contre, il y a parmi les étudiants, ceux qui ont choisi un équivalent qui n'est pas correct comme l'exemple du « Roi Fouad 1er» qu'ils ont transcrit en «Le Roi Fouad Le Premier » : ces étudiants ont calqué la structure arabe sans se rendre compte que la transcription française des noms propres suivis d'un adjectif numéral ordinal «indiquant le rang d'un élément au sein d'une série ou d'un ensemble »" ${ }^{49}$, exige l'omission de tout article défini séparant le nom propre de son désignatif numérique $\mathrm{Ce}$ dernier se présente généralement sous une forme abrégée soit en chiffres arabes ou romains suivi d'une, de deux ou de trois lettres minuscules surélevées qui correspondent aux lettres finales des adjectifs.

Le nom du vice-roi «Méhémet Ali » a, quant à lui, posé un problème de traduction aux étudiants puisque la plupart ont translitéré le nom et ont utilisé la graphie arabe «Muhammad Ali » et non la graphie turque «Méhémet Ali » couramment utilisée en français. Pour surmonter ce problème et respecter en quelque sorte la prononciation et la graphie arabe du nom, nous leur avons suggéré de conserver la graphie turque " Méhémet Ali" tout en ajoutant, entre parenthèses, à la première occurrence du nom dans le texte, la graphie arabe «Muhammad Ali ». Cette stratégie nous a permis à la fois de répondre à la demande du donneur d'ouvrage de conserver l'orthographe turque pour différencier entre «Muhammad Ali », le vice-roi et Mohammed Ali Clay ${ }^{50}$, le célèbre boxeur américain.

\subsubsection{Praxonymes : (Noms de faits historiques)}

Les praxonymes désignent les noms de faits ou d'événements historiques qui peuvent revêtir un nom différent selon le point de vue

\footnotetext{
${ }^{48} \mathrm{https://www.universalis.fr/encyclopedie/anouar-el-sadate/}$

Dernière consultation le 04/08/2020

${ }^{49}$ http://bdl.oqlf.gouv.qc.ca/bdl/gabarit_bdl.asp?id=4271

Dernière consultation le 04/08/2020

${ }^{50} \mathrm{https} / / / \mathrm{www}$. linternaute.fr/sport/biographie/1777200-mohamed-ali-biographie-

courte-dates-

citations/\#: :text=Biographie\%20courte\%20de\%20Mohamed\%20Ali\%20\%2D $\% 20$

N\%C3\%A9\%20le\%2019\%20janvier\%201942,reprises\%2C\%20sacr\%C3\%A9\%20c

hampion\%20du\%20monde
}

Dernière consultation le 04/08/2020 
du public source ou de la société de départ comme le montre 1'exemple ci-dessous :

- Exemple (1) حرب السادس من أكتوبر:

\begin{tabular}{|c|c|c|}
\hline Notre traduction & $\begin{array}{c}\text { Traduction proposée par } \\
\text { les étudiants }\end{array}$ & Texte source \\
\hline $\begin{array}{c}\text { La guerre du 6 octobre } \\
1973\end{array}$ & La guerre du Kippour & \\
\hline
\end{tabular}

La traduction du nom de la Guerre arabo-israélienne a constitué un obstacle de traduction pour les étudiants en raison de l'appellation erronée de cette guerre dans les contextes français. En fait, le nom de cette guerre y est souvent traduit par «La Guerre du Kippour » ${ }^{51}$ ou « La Guerre de Yom Kippour » en référence au jour du Grand Pardon des Israélites, jour où a eu lieu la guerre du 6 octobre 1973 et non par «La Guerre du 6 octobre » ou «La victoire du 6 octobre ». Ainsi, beaucoup d'étudiants ont traduit le nom de cette guerre comme ils l'ont trouvé dans les contextes français «La Guerre du Kippour » ou «La Guerre de Yom Kippour », ce qui est considéré comme une erreur culturelle de traduction puisque cette traduction associe cette guerre à une fête juive et lui attribue son appellation. Or, d'un point de vue arabe, cette guerre fut déclenchée le 6 octobre 1973 et en plein Ramadan, et fut une victoire pour l'armée égyptienne. Le nom qu'il faut lui attribuer serait donc « La victoire du 6 octobre » ou « La guerre du 6 octobre », ou même parfois «La guerre du dix Ramadan » afin de respecter la culture source. Nous avons donc conseillé aux étudiants d'opter pour une traduction neutre marquant uniquement la date de la guerre comme suit : « la guerre du 6 octobre 1973 ».

\subsubsection{Expressions figées :}

Selon Gross, le linguiste français, les expressions figées sont caractérisées par un figement sur le plan syntaxique ou sémantique: "une séquence est figée du point de vue syntaxique quand elle refuse toutes les possibilités combinatoires ou transformationnelles qui caractérisent habituellement une suite de ce type. Elle est figée sémantiquement quand le sens est opaque ou non compositionnel, c'est-à-dire quand il ne peut pas être déduit du sens

${ }^{51}$ https://www.cairn.info/revue-defense-nationale-2015-10-page-122.htm Dernière consultation le 05/03/2019 
$\overline{\text { des éléments composants }{ }^{52} \text {. A la majorité des études sur le }}$ figement, sont associés, entre autres, les termes suivants : expressions idiomatiques $^{53}$, collocations ${ }^{54}$, locutions ${ }^{55}$, proverbes $^{56}$, etc.

En fait, "l'utilisation des expressions figées dans le texte littéraire n'est pas sans effets sur son esthétique, puisqu'elles peuvent rendre compte d'une valeur ajoutée à travers les sonorités et la prosodie caractéristiques de ces mêmes expressions ${ }^{57}$. En plus, l'étude des expressions figées dans un texte «nous renvoie inévitablement à examiner les notions d'intertextualité et d'interculturalité. Leur inscription dans le texte [...] permet de le mettre en écho avec d'autres univers : culturels, sociaux, littéraires, etc ${ }^{58}$.

${ }^{52}$ Maurice GROSS : « Sur les phrases figées complexes du français », in Langue française 77 , pp. 50.

${ }^{53}$ Une expression idiomatique est une construction ou une locution particulière à une langue, qui porte un sens par son tout et non par chacun des mots qui la composent. Il peut s'agir de constructions grammaticales ou, le plus souvent, d'expressions imagées ou métaphoriques. Elle est en général intraduisible mot à mot, et il peut être difficile, voire impossible, de l'exprimer dans une autre langue.

http://classeelementaire.free.fr/maitrise-

langue/vocabulaire/activites/expressions/definition-idiomatique.pdf

Dernière consultation le 09/02/2020

${ }^{54}$ Les collocations sont des associations lexicales privilégiées et sémantiquement compositionnelles.

Agnès TUTIN : « Les collocations lexicales : une relation essentiellement binaire

définie par la relation prédicat-argument », in Langages , $\mathrm{n}^{\circ} 189,2013$, page 47.

${ }^{55}$ Les locutions sont des expressions idiomatiques, des expressions qui doivent être apprises telles quelles lorsque l'on acquiert une langue première ou une langue seconde. Outre son caractère figé, la locution a la propriété d'être une unité autonome, qui appartient à l'une ou l'autre des grandes classes de mots.

https://www.erudit.org/fr/revues/qf/2014-n171-qf01221/71229ac.pdf

Dernière consultation le 09/02/2020

${ }^{56}$ Le proverbe est une sentence courte et imagée, d'usage commun, qui exprime une vérité d'expérience ou un conseil de sagesse et auquel se réfère le locuteur.

https://www.cnrtl.fr/definition/proverbe

Dernière consultation le 09/02/2020

${ }^{57}$ Céline GUILLEUX: «Les expressions figées : analyse linguistique et littéraire et exploitation en classe de français langue étrangère (FLE) », in, Calenda, Publié le mercredi 25 juillet 2018.

https://calenda.org/461019

Dernière consultation lé $10 / 8 / 2020$

${ }^{58}$ Ibid.,Loc.cit. 
Or, si la traduction interlinguale pose des problèmes du simple fait de la présence de deux systèmes linguistiques différents, qu'en est-il pour la traduction des expressions figées ? A vrai dire, la traduction des expressions figées est encore plus compliquée et moins évidente, notamment entre des langues n'ayant pas le même patrimoine historico-culturel. «La traduction des expressions figées de l'arabe vers le français dépasse ainsi les problèmes dus aux différences de catégorisation et de grammaticalisation entre ces langues, le figement étant un mécanisme cristallisant l'idiomaticité d'une langue » 59

La traduction de ces expressions suit donc la même démarche que toute traduction humaine impliquant la mise en œuvre d'un « processus cognitif» qui «consiste pour le traducteur à comprendre un texte/discours pour le faire comprendre à des destinataires qui $n$ 'ont pas accès à l'original » 60

Dans le corpus étudié, les étudiants avaient relevé quelques expressions figées et leur avaient proposé des traductions. Ces traductions n'étaient pas souvent adéquates, du fait que certains étudiants n'avaient pas saisi la globalité sémantique de ces expressions, c'est-à-dire qu'ils n'avaient pas compris que le sens global de ces expressions ne provient pas du sens des unités lexicales qui les composent puisqu'elles sont des segments noncompositionnels qui cachent souvent un aspect métaphorique ${ }^{61}$.

- Exemple (8): فى نهايت المطاف

\begin{tabular}{|c|c|c|}
\hline Notre traduction & $\begin{array}{c}\text { Traduction proposée par } \\
\text { les étudiants }\end{array}$ & Texte source \\
\hline $\begin{array}{c}\text { En fin de compte } \\
\text { Finalement }\end{array}$ & A la fin de la tournée & \\
\hline
\end{tabular}

Cette expression figée signifie «finalement» ou «en fin de compte ». La traduction littérale de cette expression figée, comme elle fut proposée par certains étudiants «à la fin de la tournée », va aboutir

59 Salah MEJRI :«Figement et traduction: problématique générale », in M3TA, n.53, 2008, p. 245.

${ }^{60}$ Christine DURIEUX : « Mettre la main sur le figement lexical : la démarche du traducteur », in Meta, 53, 2008, p. 324.

${ }^{61}$ Cf. Mohamed SAAD ALI : "La traduction des expressions figées : langue et culture", in Traduire, 235 | 2016, pp. 103-123. 
à une expression incompréhensible pour le public cible. En effet, l'origine dérive des pratiques musulmanes et signifie l'endroit où le pèlerin accomplit ses sept tours autour de la Ka'ba dans le sens inverse des aiguilles d'une montre.

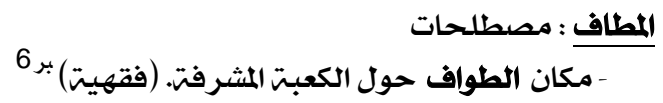

Or, cette expression d'origine religieuse a acquis un sens figuré au cours des années pour signifier en arabe : «à la fin, après tout, au bout du compte, cependant, dans tous les cas, de toute façon, de toute manière, en définitive, enfin, en tout cas, finalement, malgré tout, pourtant, quand même, seulement, tout de même, toutefois, ultimement, en finale ${ }^{63}$. Ainsi, la locution adverbiale " en fin de compte» ou l'adverbe «finalement» sont utilisés pour exprimer le même sens. L'utilisation de l'équivalence fonctionnelle de cette expression permet l'intelligibilité de cette expression figée dans la langue cible.

- Exemple ( 9 ) : لابد من صنعا وإن طال السفر

\begin{tabular}{|c|c|c|}
\hline Notre traduction & $\begin{array}{c}\text { Traduction proposée par } \\
\text { les étudiants }\end{array}$ & Texte source \\
\hline $\begin{array}{c}\text { Persévérance mène à } \\
\text { récompense }\end{array}$ & Cela vaut la peine & $\begin{array}{l}\text { لابد من صنعرا وإن طال } \\
\text { السفر }\end{array}$ \\
\hline
\end{tabular}

La traduction de l'expression idiomatique "لابد من صنعًا وإن طال السفر ", qui n'est pas une expression courante même en arabe, a constitué un obstacle de traduction devant les étudiants car cette expression appartient au patrimoine culturel islamique. Elle fut mentionnée par l'imam Ahmad Ibn Hanbal, éminent savant du hadîth et fondateur de l'école hanbalite, l'une des quatre principales écoles juridiques du monde musulman d'aujourd'hui. De plus, Abdel Aziz Al Maqualeh, le poète yéménite notoire, l'avait emprunté pour composer le premier

\footnotetext{
${ }^{62} \mathrm{https} / / / \mathrm{www} . a l m a a n y . c o m / a r / d i c t / a r-$ ar/\%D8\%A7\%D9\%84\%D9\%85\%D8\%B7\%D8\%A7\%D9\%81/

Dernière consultation le 08/09/2020

${ }^{63}$ Dictionnaire Sensagent en ligne http://dictionnaire.sensagent.leparisien.fr/en\%20fin\%20de\%20compte/fr-fr/ Dernière consultation le 08/09/2020
} 
vers de son poème éponyme célèbre à l'imam Ahmad Ibn Hanbal, pour constituer le titre et le premier vers d'un poème composé par ${ }^{64}$.

Cette expression a une histoire : il y a treize siècles, l'imam Ahmad bin Hanbal, quitta l'Irak pour se rendre au Yémen, et précisément à Sanaa pour rencontrer l'imam Abdul Razzaq Al-San'ani ${ }^{65}$., érudit sunnite islamique de la science des hadiths et l'auteur du célèbre ouvrage AL-MOUSSANNAF ${ }^{66}$, et profiter de son large savoir. En route pour le Yémen, il s'arrêta à la Mecque et là, il sut que l'Imam alSan'ani était en pèlerinage. On lui dit alors que la présence du grand imam en ces lieux saints allait lui épargner le voyage pour le Yémen. Cependant, Ahmad bin Hanbal refusa et préféra se rendre à Sanaa pour rencontrer l'Imam al-San'ani dans son lieu de résidence et il prononça sa célèbre expression : لابد من صنعًاء وإِن طال السفر qui devient plus tard : لابد من صنعًا وإن طال السفر qui signifie que la peine endurée dans son voyage jusqu'à Sanaa sera récompensée par la rencontre de l'imam Abdul Razzaq Al-San'ani. Cette expression fut utilisée dès lors en arabe pour signifier que tout effort sera en fin de compte récompensé. Pour traduire cette expression idiomatique, nous avons suggéré aux étudiants d'avoir recours à un proverbe équivalent : « Persévérance mène à récompense ».

\section{- Exemple 10 (الليالي الملاح: 10}

\begin{tabular}{|c|c|c|}
\hline Notre traduction & $\begin{array}{c}\text { Traduction proposée par } \\
\text { les étudiants }\end{array}$ & Texte source \\
\hline Les grands jours & $\begin{array}{c}\text { Les belles nuits/ } \\
\text { Les beaux jours }\end{array}$ & الليالي الملالاحلي \\
\hline
\end{tabular}

${ }^{64}$ Cf., https://almoheet.net/\%D8\%B4\%D8\%B9\%D8\%B1-

$\% \mathrm{D} 8 \% \mathrm{~B} 9 \% \mathrm{D} 8 \% \mathrm{~A} 8 \% \mathrm{D} 8 \% \mathrm{AF}-$

\%D8\%A7\%D9\%84\%D8\%B9\%D8\%B2\%D9\%8A\%D8\%B2-

\%D8\%A7\%D9\%84\%D9\%85\%D9\%82\%D8\%A7\%D9\%84\%D8\%AD/

Dernière consultation le $08 / 09 / 2020$

${ }^{65} \mathrm{Cf}$. https://almoslim.net/node/241806 pour l'histoire en arabe de cette expression.

Dernière consultation le $08 / 09 / 2020$

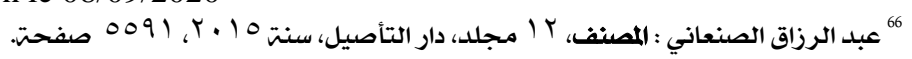


L'expression "الليالي الملاح " est également une forme d'expression figée, nommée collocation. Cette expression arabe est une expression ancienne qui fut utilisée dans le passé pour décrire les célébrations qui pouvaient durer plusieurs jours comme les mariages ou les couronnements royaux. En fait, la traduction littérale suggérée par certains étudiants «Les beaux jours » ou «Les belles nuits » est une traduction correcte sur le plan sémantique puisque ces jours sont des jours caractérisés par la beauté des célébrations, de la musique, des costumes, des danses, etc. Or, nous avons opté pour l'expression équivalente "les grands jours », puisque c'est une expression française utilisée pour décrire les jours de grandes célébrations comme les jours de mariage, ou les jours de remise des diplômes. Cette expression «les grands jours » utilisée en français a aussi une origine ancienne car celle-ci désignait dans le passé «des assises extraordinaires d'une délégation du Parlement, tenues dans certaines villes, au Moyen Âge et jusqu'à la fin de l'Ancien Régime, pour juger en appel des affaires jugées en premier ressort par les magistrats locaux, ou en premier et dernier ressort certaines affaires exceptionnelles ou certains coupables auxquels leur rang ou leur fortune eussent pu assurer l'impunité auprès des juges du pays $\gg{ }^{67}$.

Le traducteur doit donc comprendre la situation d'usage d'une expression dans la langue de départ pour lui trouver l'expression appropriée équivalente utilisée dans la même situation.

\subsection{Problèmes socioculturels:}

Il s'agit des éléments qui se lient particulièrement à la culture de départ. Du point de vue de la traduction, ils se caractérisent par leur spécificité culturelle qui suscite ensuite des problèmes traductologiques. Les problèmes socioculturels dans le corpus étudié recouvrent surtout les notions religieuses, les notions historiques comme les grades militaires, les titres de noblesse, les noms de ministères et des systèmes éducatifs, etc.

${ }^{67}$ Dictionnaire Larousse en ligne https://www.larousse.fr/encyclopedie/divers/Grands_Jours/180024 Dernière consultation le 09/07/2021 


\subsubsection{Notions religieuses:}

Ce sont des éléments référant à des notions religieuses connues uniquement dans la culture de départ. Ces mots constituent un problème en traduction devant tout traducteur. Dans le corpus en question, la traduction de notions religieuses ou de titres religieux propres à la culture musulmane constituait un problème à cause de l'absence, souvent, d'équivalent dans la culture cible. Pour résoudre ce problème, nous avons utilisé des mots provenant d'emprunts.

- Exemple (1): الوهابية / الوهابيون:

\begin{tabular}{|c|c|c|}
\hline Notre traduction & $\begin{array}{c}\text { Traduction proposée par } \\
\text { les étudiants }\end{array}$ & Texte source \\
\hline $\begin{array}{c}\text { L'entrée de l'Égypte en } \\
\text { guerre contre le } \\
\text { wahhabisme. (Le } 3 \text { janvier } \\
\text { 1811). } \\
\text { En 1815, Tosson conclut } \\
\text { une trêve avec les } \\
\text { wahhabites, en vertu de } \\
\text { laquelle ils maintinrent } \\
\text { l'autorité sur le Nejd et } \\
\text { certaines régions du } \\
\text { Hedjaz. }\end{array}$ & $\begin{array}{c}\text { L'entrée de l'Égypte en } \\
\text { guerre contre le courant } \\
\text { wahhabiste. (Le } 3 \text { janvier } \\
\text { 1811). } \\
\text { En 1815, Tosson conclut } \\
\text { une trêve avec les } \\
\text { wahhabites, en vertu de } \\
\text { laquelle ils ont maintenu } \\
\text { l'autorité sur le Nejd et } \\
\text { certaines régions du } \\
\text { Hedjaz. }\end{array}$ & 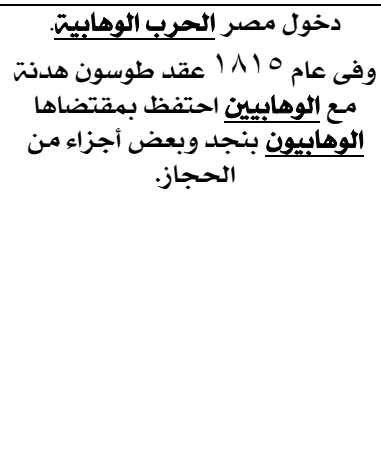 \\
\hline
\end{tabular}

Dans le dictionnaire Almaani, le mot (وَهَّابيّة) signifie la doctrine islamique fondée par le cheikh Mohamed ibn Abdel wahab au XVIII

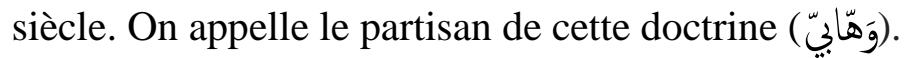

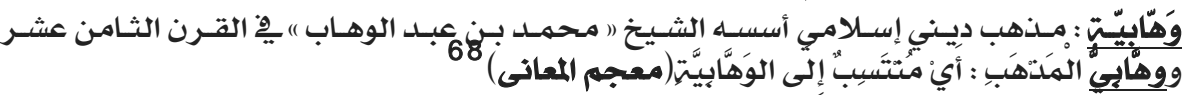

Pour traduire le mot (الوهابية), les étudiants avaient suggéré (le courant wahhabiste). Cependant, nous avons suggéré aux étudiants d'utiliser (wahhab) comme base anthroponymique, puis d'ajouter le suffixe (isme) qui sert à former un nom masculin correspondant à une doctrine, pour obtenir enfin (wahhabisme). En fait, selon la définition

${ }^{68}$ Dictionnaire Almaany en ligne

www.almaany.com,

Dernière consultation le 19 mai 2018. 
du Centre collégial de développement de matériel didactique au Québec (CCDMD) ${ }^{69}$ :

"On confond souvent les finales -isme ou -iste. Il faut faire le bon choix, car ces suffixes servent à former des mots tout à fait différents. Les erreurs les plus nombreuses proviennent de la confusion des finales -isme et -iste. Pour contrer cette difficulté, vous retiendrez : que les noms de personnes n'ont jamais de suffixe en-isme; que le suffixe -iste sert à former des noms de personnes correspondant aux noms en isme et ne forme jamais de noms exprimant une théorie (ou doctrine) ; que le suffixe -iste sert aussi à former certains adjectifs qui indiquent une relation $»$.

La définition du «wahhabisme» fut également donnée par l'orientaliste Henri Laoust comme suit : «Mouvement à la fois religieux et politique, arabe et musulman, le wahhābisme s'est assigné essentiellement pour but [...] de construire un État sunnite qui se fût étendu non seulement au Nejd mais à l'ensemble des pays arabes, de restaurer l'Islam dans sa pureté première...» ${ }^{\mathbf{7 0}}$

${ }^{69}$ Le Centre collégial de développement de matériel didactique (CCDMD), géré par le collège de Maisonneuve, est un centre de production de ressources numériques et de documents imprimés conçus à l'intention du personnel enseignant et des étudiants de l'ensemble du réseau collégial du Québec.

${ }^{70}$ «WAHHĀBISME », Encyclopædia Universalis [en ligne],. : http://www.universalis.fr/encyclopedie/wahhabisme/ Dictionnaire de l'Islam (religion et civilisation) publié par EncyclopædiaUniversalis.

Dernière consultation le 18 mai 2017 
De même, pour traduire le mot (وَهّابيون), nous avons expliqué aux étudiants qu'ils devaient d'abord utiliser (wahhab) en tant que base anthroponymique, ensuite lui ajouter le suffixe $(-i t e)^{71}$ qui sert à former des substantifs désignant les adeptes d'une personne ou les partisans d'une doctrine. D'ailleurs, la définition du partisan de cette doctrine nous a été donnée par Jean-Pierre Lacroux :

"Que sa dénomination dérive d'un nom commun ou d'un nom propre, l'adepte ne prend jamais de majuscule initiale. L'adhérent, le disciple, le fidèle, le membre pas davantage. Toutes les catégories sont concernées (religion, philosophie, littérature, arts, politique, etc.): un anglican, sept bouddhistes, trois catholiques, [...], un wahhabite, un

- Exemple (3) : (إمام)

\begin{tabular}{|c|c|c|}
\hline Notre traduction & $\begin{array}{c}\text { Traduction proposée par } \\
\text { les étudiants }\end{array}$ & Texte source \\
\hline Imâm & $\begin{array}{c}\text { Chef de la prière chez les } \\
\text { musulmans }\end{array}$ & إمام \\
\hline
\end{tabular}

Dans l'exemple ci-dessus, Les étudiants avaient utilisé à la place du mot (imâm), la définition suivante «le chef de la prière »en s'inspirant des différentes définitions présentes dans les dictionnaires en ligne à ce sujet ${ }^{73}$. Nous avons donc expliqué aux étudiants que le

${ }^{71}$ «-ite », dans TLFi, Le Trésor de la langue française informatisé, dernière consultation le 19 mai 2017.

${ }^{72}$ Jean-Pierre LACROUX, Orthotypographie : Orthographe \& typographie françaises : Dictionnaire raisonné, 2007), in http://www.orthotypographie.fr/ Dernière consultation le 18 /05/2019..

${ }^{73} \mathrm{https} / / / \mathrm{cnrtl}$.fr/definition/imam

Dernière consultation le 19/06/ 2019 
mot emprunté à l'arabe (imâm), est utilisé fréquemment en français pour désigner «le chef de la prière dans une mosquée ». De plus, les médias en France utilisent aujourd'hui le titre « le grand imam de la mosquée d'Al-Azhar ${ }^{74}$. En outre, le mot imâm a été introduit dans les dictionnaires français et est définit comme suit par le Dictionnaire de l'Académie Française: « Imam, n.m : Emprunté de l'arabe (imâm), dérivé de (amma), chercher en tête ou présider. Titre donné à différents dignitaires de la religion musulmane. Mahomet fut le premier iman. Le rôle essentiel de l'imam est de présider la prière $»^{\mathbf{7 5}}$ Ainsi, nous avons opté pour le maintien de l'appellation originale afin de conserver le plus grand nombre des culturèmes.

\subsubsection{Notions historiques :}

Il s'agit de termes ou de notions qui furent utilisés à une certaine époque de l'histoire de l'Égypte mais qui maintenant n'existent plus en raison de la disparition de ce qu'ils représentent. Ces notions historiques peuvent être des établissements, des grades militaires, des titres de noblesse, des systèmes éducatifs, des noms de ministères, etc. En fait, ces différents éléments révolus sont la preuve de l'évolution de notre société tout comme les mots qui disparaissent du Dictionnaire. Bernard Cerquiglini, éminent linguiste et professeur à l'université Paris Diderot le rappelle en disant : «les noms de métiers, par exemple, connaissent toujours un important renouvellement: les chaufourniers, coffretiers et argenteurs ont peu à peu laissé place aux informaticiens, développeurs et collaborateurs en tous genres $»^{76}$.

${ }^{74} \mathrm{https}: / / \mathrm{www}$. lepoint.fr/societe/le-grand-imam-d-al-azhar-l-homme-qui-detient-lescles-de-1-islam-17-06-2016-2047402_23.php

Dernière consultation le 19/05/ 2019.

${ }^{75}$ Dictionnaire de l'Académie française, neuvième édition, version informatisée, dernière consultation le 19 mai 2019.

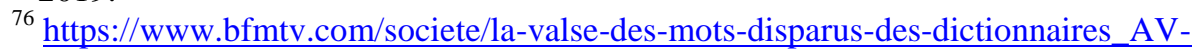
201811150079.html

Dernière consultation le 18 /07/2019 


\subsubsection{Des appellations de gouvernements :}

- Exemple (1) : (الباب العالى)

\begin{tabular}{|c|c|c|}
\hline Notre traduction & $\begin{array}{c}\text { Traduction proposée par } \\
\text { les étudiants }\end{array}$ & Texte source \\
\hline $\begin{array}{c}\text { La Sublime Porte (surnom } \\
\text { du gouvernement } \\
\text { ottoman) }\end{array}$ & Le gouvernement ottoman & \\
\hline
\end{tabular}

En consultant le dictionnaire en ligne ALmaany, nous avons trouvé que le terme الباب العالىsignifie le gouvernement ottoman.

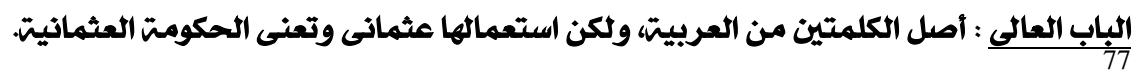

Le site universalis.fr, nous fournit également une explication détaillée de l'évolution de ce terme :

$$
\text { «Le mot }
$$

turc kapi désigne une porte, mais aussi le palais du sultan (d'où parfois l'expression «porte ottomane»), puis le palais du grand vizir et enfin le siège $d u$ gouvernement. Les troupes du sultan étaient désignées par l'expression kapi kullari, c'est-à-dire "les esclaves de la Porte ». À partir de 1654, le grand vizir fut doté d'un palais particulier où se tinrent les séances du divan ou

77 Dictionnaire Almaany"en ligne, www.almaany.com, dernière consultation le 19/09/2019. 
gouvernement; ce palais fut d'abord appelé Pasa kapisi (palais du Pacha), puis Bab-i Âli, la "Sublime Porte», nom sous lequel les Occidentaux ont englobé à la fois le palais du sultan, la cour ottomane, le gouvernement et finalement l'État ottoman lui-même $»^{78}$.

Ce texte étant à vocation culturelle, il est important donc de conserver le plus grand nombre de culturèmes possible. Dans ce but, nous avons expliqué aux étudiants que la traduction de الباب العالي en «Sublime porte » serait plus concordante au contexte . Nous leur avions proposé d'utiliser alors une combinaison de procédés de traduction pour mieux transmettre le sens: L'équivalence et l'étoffement. À la première occurrence du terme (Al Bâb Al 'ali) dans le texte, nous avons utilisé le terme équivalent (la Sublime Porte), suivi d'une explication entre parenthèses. Grâce à cette stratégie, nous avons expliqué au public cible la signification de cette appellation.

\subsubsection{Des établissements et des postes :}

مدرسة (دار العلوم) : Exemple (1)

\begin{tabular}{|c|c|c|}
\hline Notre traduction & $\begin{array}{c}\text { Traduction proposée par } \\
\text { les étudiants }\end{array}$ & Texte source \\
\hline $\begin{array}{c}\text { L'école Supérieure } \\
\text { (Dar al-O'lūm). }\end{array}$ & L'Ecole des Sciences & \\
\hline
\end{tabular}

La traduction de مدرست (دار العلوم) a constitué une difficulté de traduction chez les étudiants et a abouti à une erreur de traduction puisque les étudiants l'ont traduit en «l'Ecole des Sciences » croyant donner ainsi

${ }^{78}$ https://www.universalis.fr/encyclopedie/porte-sublime-porte/ Dernière consultation le $20 / 07 / 2020$ 
l'équivalent de l'appellation arabe de cette école. Or, il fallait se référer au contexte culturel égyptien de l'époque, c'est-à-dire aux années 1871, date de la création de cette école, pour comprendre d'abord que le mot 《دمرست la fois, école et faculté. Aussi, (دار العلوم) n'était pas une école qui enseignait les sciences comme l'ont suggéré certains étudiants, mais une Grande Ecole qui enseignait, entre autres, la jurisprudence, les sciences littéraires, l'histoire générale, la géographie, l'arithmétique, la géométrie, la chimie, la physique et la calligraphie arabe ${ }^{79}$. La compréhension du groupe de mots مدرست (دار العلوم) dans son contexte, exigeait alors une compréhension générale du système éducatif égyptien à l'époque.

Nous avons donc utilisé deux procédés de traduction : l'adaptation et la translitération et suggéré aux étudiants la traduction suivante: L'école Supérieure (Dar al-O'lūm). Cette traduction transmet bien le message voulu et rend aisée son intelligibilité car la traduction suggérée par les étudiants de (دار العلوم)en (l'Ecole des Sciences), peut induire le lecteur en erreur.

- Exemple (2) وزارة محمد محمود الثالثت:

\begin{tabular}{|c|c|c|}
\hline Notre traduction & $\begin{array}{c}\text { Traduction proposée par } \\
\text { les étudiants }\end{array}$ & Texte source \\
\hline Le troisième & Le ministère de & Mohamed Mahmoud \\
guhamernement de & Mounad Mahmūd & \\
& & \\
\hline
\end{tabular}

${ }^{79}$ https://gate.ahram.org.eg/daily/News/131747/121/458256/\%D8\%AA\%D8\%B1\%D8\%A7\% D8\%AB-\%D9\%88\%D8\%AD\%D8\%B6\%D8\%A7\%D8\%B1\%D8\%A9/\%D8\%AD\%D9\%83\%D8\%A7\%D9\%8A \%D8\%A9-\%D8\%AF\%D8\%A7\%D8\%B1\%D8\%A7\%D9\%84\%D8\%B9\%D9\%84\%D9\%80\%D9\%80\%D9\%88\%D9\%85.aspx Dernière consultation le 03/05/2019 


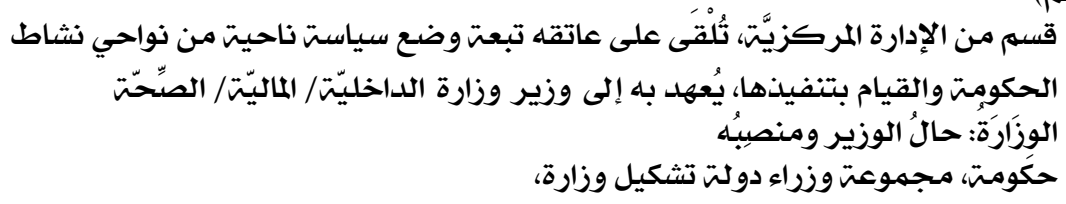

Dans les dictionnaires arabes, le mot "وزارة " est un mot polysémique qui peut signifier à la fois «ministère » et « gouvernement » comme le montrent les définitions ci-dessus. Pour comprendre quel sens choisir, il fallait faire des recherches et découvrir que le sens du mot " وزارة " dans ce contexte voulait dire « gouvernement » et non «ministère » comme l'ont suggéré certains étudiants. En effet, une mauvaise compréhension du mot hors-contexte a entrâné un faux- sens considéré comme une grave erreur en traduction puisque Muhamad Mahmūd n'était pas un ministre qui présidait un Ministère mais un Premier Ministre à la tête d'un gouvernement pour quatre mandats différents : le premier gouvernement qu'il a présidé était à l'époque du roi Fouad I et a duré du 27 juin 1928 au 4 octobre 1929; le deuxième gouvernement, était à l'époque du roi Farouk et a duré du 30 décembre 1937 au 27 avril 1938 ; le troisième gouvernement a duré du 27 av ril au 24 juin de la même année; Le quatrième et dernier gouvernement était du 24 juin 1938 au 18 août $1939^{81}$. Ainsi, nous avons opté pour « le troisième gouvernement de Muhamad Mahmūd » comme traduction à cette expression.

\section{- Exemple (3) مدير عام مصلحت المساحتر}

\begin{tabular}{|c|c|c|}
\hline Notre traduction & $\begin{array}{c}\text { Traduction proposée par } \\
\text { les étudiants }\end{array}$ & Texte source \\
\hline $\begin{array}{c}\text { Directeur Général du } \\
\text { Cadastre }\end{array}$ & $\begin{array}{c}\text { Directeur général de la } \\
\text { surface/ Directeur } \\
\text { général de la Mesaha. }\end{array}$ & \\
\hline
\end{tabular}

${ }^{80}$ Dictionnaire Almaany en ligne https://www.almaany.com/ar/dict/arar/\%D9\%88\%D8\%B2\%D8\%A7\%D8\%B1\%D8\%A9/

Dernière consultation le $03 / 05 / 2019$

${ }^{81}$ https://www.marefa.org/\%D9\%85\%D8\%AD\%D9\%85\%D8\%AF_\%D9\%85\%D8 \%AD\%D9\%85\%D9\%88\%D8\%AF_\%D8\%A8\%D8\%A7\% D8\%B4\%D8\%A7

Dernière consultation le 03/06/2019 
La traduction de مصلحت المساحت dans مدير عام مصلحت المساحت constituait également un problème de traduction pour les étudiants puisque cette «Direction » construite en 1898, sous le nom de مصلحت المساحت العموميت n'existe plus en Egypte ; ou autrement dit, existe actuellement sous un autre nom الهيئت المصريت العامتة للمساحتَ ${ }^{82}$. Il fallait donc trouver un équivalent français à cette Direction qui joue presque le même rôle. Nous avons alors conseillé aux étudiants d'effectuer des recherches documentaires pour trouver l'organisme correspondant. Le Cadastre ou La Direction du Cadastre, de la Conservation Foncière et de 1'Enregistrement (DCCFE) ${ }^{83}$ fut l'Organisme correspondant à مصلحتَ dont la mission est de faire «l'inventaire exhaustif et permanent, descriptif et évaluatif de la propriété foncière, qu'il s'agisse des parcelles de terrain, ou des locaux (missions individuelles, appartements, commerce, usines, ...) ${ }^{84}$. La traduction du syntagme مدير عام مصلحت المساحت devient donc : "Directeur Général du Cadastre ».

\subsubsection{Des Systèmes éducatifs :}

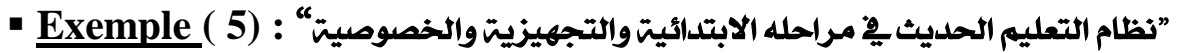

\begin{tabular}{|c|c|c|}
\hline Notre traduction & $\begin{array}{c}\text { Traduction proposée par } \\
\text { les étudiants }\end{array}$ & Texte source \\
\hline $\begin{array}{c}\text { Le système éducatif } \\
\text { moderne dans ses cycles : } \\
\text { primaire, préparatoire et } \\
\text { spécialisé (qui correspond à } \\
\text { l'enseignement primaire, } \\
\text { secondaire et } \\
\text { supérieur français). }\end{array}$ & $\begin{array}{l}\text { Le système des cycles } \\
\text { primaires, préparatoires } \\
\text { ainsi que le système privé }\end{array}$ & 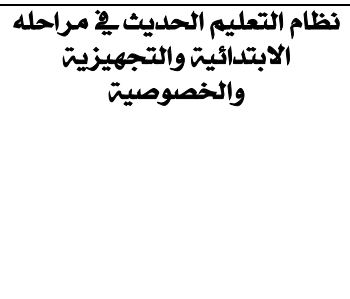 \\
\hline
\end{tabular}

\footnotetext{
${ }^{82}$ http://www.esa.gov.eg/history.aspx

Dernière consultation le 06/06/2019

${ }^{83} \mathrm{http}: / /$ www.dadc.gouv.tg/index.php/direction/vision-missions.html

Dernière consultation le 06/06/2019

${ }^{84}$ Ibid., Loc.cit

Dernière consultation le 06/06/2019
} 
La traduction de " "ظام التعليم الحديث في مراحل الابتدائية والتجهيزة والحصوصية fut un obstacle de traduction qui se dressa devant les étudiants parce que les différents cycles qui constituent ce système ne figurent plus dans le système éducatif égyptien actuel et il fallait comprendre la signification de chacun de ces cycles. Certains étudiants n'avaient pas réussi à trouver la traduction correspondante et ils avaient traduit cette expression en «le système des cycles primaires, préparatoires ainsi que le système privé »; d'autres étudiants avaient essayé de trouver l'équivalent français adéquat à ces différents cycles dans le système éducatif français actuel pour que la traduction soit compréhensible et l'avaient traduit comme suit: «l'enseignement primaire, secondaire et supérieur ${ }^{85}$.

Or, ces deux solutions ne pouvaient pas être acceptées, puisque la première suggestion ne correspondait pas à la réalité du système éducatif à l'époque de Méhémet Ali qui était sectorisé en plusieurs cycles «primaire, préparatoire et spécialisé ${ }^{86}$. Le cycle primaire, d'une durée de 3 ans, accueillait les apprenants qui avaient entre 7 et 12 ans pour les préparer au niveau préparatoire qui durait 4 ans. Après ces deux cycles, les apprenants étaient prêts à intégrer le cycle spécialisé qui comprenait les grandes écoles spéciales ou spécialisées comme l'Ecole de Médecine, l'Ecole Polytechnique, l'Ecole de Musique, l'Ecole de commerce, etc.

La seconde suggestion basée sur l'équivalence culturelle ne pouvait également pas être acceptée puisqu'il fallait être fidèle au contexte source et puisqu'il y avait maints contextes en langue française qui parlaient du système éducatif à l'époque de Méhémét Ali ${ }^{87}$.

Nous avons donc suggéré aux étudiants de recourir à deux procédés : la traduction littérale et l'équivalence; autrement dit, de traduire littéralement les différents cycles du système éducatif égyptien à l'époque de Méhémet-Ali et de faire un ajout explicatif entre

${ }^{85} \mathrm{https}: / / \mathrm{www}$. france-education-international.fr/article/le-systeme-educatiffrancais?langue $=\mathrm{fr}$

Dernière consultation le 05/05/2019

${ }^{86}$ Cf. Elena AMBROSETTI: Egypte, l'exception démographique, Coll. Les Cahiers de l'INED, éds. de l'INED, p.94.

${ }^{87}$ Cf . Pierre Nicolas HAMONT : L'Egypte, sous Méhémet-Ali, Léautey et Lecointe, Paris, 1843. 
parenthèses qui cite l'équivalent français pour aboutir à la traduction suivante: Le système éducatif moderne dans ses cycles : primaire, préparatoire et spécialisé (qui correspond à l'enseignement primaire, secondaire et supérieur français).

\subsubsection{Des anciens titres de noblesse et des grades militaires:}

«Les titres sont des dénominations qui renvoient au rang, à la dignité, la formation, la charge ou au grade de leur porteur. Utilisés surtout dans la communication écrite, comme formules d'appel, ils sont accompagnés de prédicats honorifiques. Ils peuvent se référer à des individus ou à des entités étatiques et exprimer une relation de subordination, d'égalité ou de supériorité entre l'expéditeur et le destinataire $\gg^{88}$.

Dans le corpus étudié, l'emploi des titres honorifiques ou des grades militaires empruntés au turc et au persan, constituait un problème de traduction. La question qui se posait était la suivante : est-ce qu'il fallait adapter ces titres en utilisant leurs équivalents dans la culture cible ou maintenir ces titres empruntés tels qu'ils sont?

\section{- Exemple (1) : (عطوفتلو أفندم)}

\begin{tabular}{|c|c|c|}
\hline Notre traduction & $\begin{array}{c}\text { Traduction proposée par les } \\
\text { étudiants }\end{array}$ & Texte source \\
\hline $\begin{array}{l}\text { Un rapport présenté } \\
\text { par Son Excellence } \\
\text { le docteur Salem } \\
\text { Pacha Salem à Son } \\
\text { Excellence, le } \\
\text { Président du conseil } \\
\text { consultatif, } \\
\text { expliquant la } \\
\text { maladie du Khédive } \\
\text { Tawfiq. }\end{array}$ & $\begin{array}{l}\text { Première proposition : } \\
\text { Un rapport présenté par Son } \\
\text { Excellence le docteur Salem } \\
\text { Pacha Salem à Sa Majesté, le } \\
\text { Président du conseil consultatif, } \\
\text { expliquant la maladie du } \\
\text { khédive Tawfiq. } \\
\text { Deuxième proposition : } \\
\text { Un rapport présenté par Son } \\
\text { Excellence le docteur Salem } \\
\text { Pacha Salem à Son Eminence le } \\
\text { Président du conseil consultatif, } \\
\text { expliquant la maladie du } \\
\text { khédive Tawfiq. }\end{array}$ & 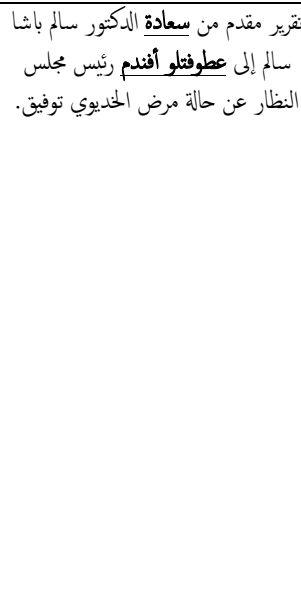 \\
\hline
\end{tabular}

${ }^{88}$ Nadine WEBER: «Titres honorifiques », in https://hls-dhs-

dss.ch/fr/articles/017435/2013-12-18/

Dernière consultation le 02/05/2019 
L'emploi de quelques titres honorifiques turcs qui n'existent plus en arabe, comme le titre 《عطوفتلو أفندم》 a constitué un problème de traduction pour les étudiants. Certains l'ont traduit «sa Majesté », d'autres 1'ont traduit « Son Eminence ». Or, les deux traductions proposées au titre "عطوفتلو آفندم " ne sont pas correctes puisque le titre « Sa Majesté » et «Son Eminence» sont des titres honorifiques associés aux rois et aux reines uniquement ${ }^{89}$. Le titre عطوفنلو أفندم est un titre turc donné aux personnes de haut rang dans la société comme marque de respect. Ainsi, ce titre fut généralement attribué aux représentants du Khédive ${ }^{90}$, au représentant d'une province ottomane $^{91}$, aux personnes occupant un poste considérable dans un ministère ou une institution ${ }^{92}$. Ce titre n'a pas un équivalent littéral dans la langue cible. Nous avons donc proposé aux étudiants d'employer le mot «Son Excellence » qui transmet le sens général de 《عطوفنلو. Nous avons également fait un allègement en omettant la traduction du mot 《 أفندم « pour éviter la redondance car ce mot sera également traduit par «Son excellence»

${ }^{89} \mathrm{https}: / / \mathrm{www}$. btb.termiumplus.gc.ca/tpv2guides/guides/clefsfp/index-

fra.html?lang=fra\&lettr=indx_catlog_m\&page=9mjwEDiH0dVs.html

Dernière consultation le 05/03/2019

${ }^{90} \mathrm{https} / / /$ al-maktaba.org/book/31917/169

Dernière consultation le 05/03/2019

${ }^{91} \mathrm{https}: / / j$ rayed.org/ar/newspapers/falastin/1911/09/02/01/article/2/?e=-------ar-20--

1--img-txIN\% 7ctxTI--------------1

Dernière consultation le 09/05/2019

92

https://gate.ahram.org.eg/daily/News/51463/86/361235/\%D9\%85\%D8\%B1\%D8\%A 7\%D9\%8A\%D8\%A7/\%D8\%AA\%D8\%B9\%D9\%8A\%D9\%8A\%D9\%86-

\%D9\%83\%D8\%A7\%D8\%AA\%D8\%A8-

\%D8\%A8\%D9\%85\%D8\%A7\%D9\%87\%D9\%8A\%D8\%A9-\%D9\%A2-

\%D8\%AC\%D9\%86\%D9\%8A\%D9\%87-

\%D8\%B4\%D9\%87\%D8\%B1\%D9\%8A\%D8\%A7\%D9\%8B-

\%D9\%84\%D9\%85\%D8\%AA\%D8\%A7\%D8\%A8\%D8\%B9\%D8\%A9-

\%D8\%A7\%D9\%84\%D8\%AD\%D8\%A7\%D9\%84\%D8\%A9-

\%D8\%A7\%D9\%84\%D8\%B5.aspx 
- Exemple (2): افندى

\begin{tabular}{|c|c|c|}
\hline Notre traduction & $\begin{array}{c}\text { Traduction proposée par } \\
\text { les étudiants }\end{array}$ & Texte source \\
\hline Effendi/ Efendi & Maître / Seigneur & أفندى \\
\hline
\end{tabular}

En fait, «effendi/ éfendi » signifie : maître chez les Turcs (titre généralement réservé aux fonctionnaires civils, aux ministres du culte, aux personnes instruites) ${ }^{\mathbf{9 3}}$. Aujourd'hui, le mot (Effendi / Efendi) est intégré dans les dictionnaires français avec ces deux variantes orthographiques.

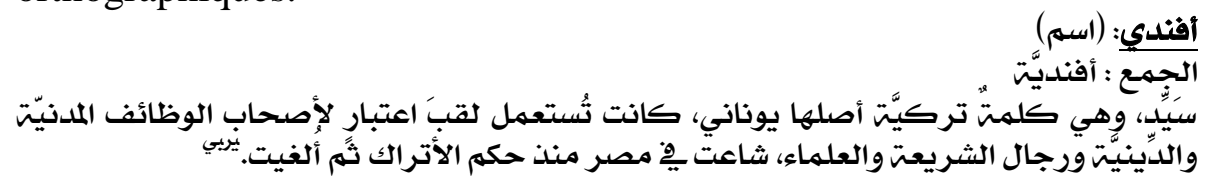

Les étudiants avaient traduit le titre «Effendi » en «Seigneur » ou «Maître » par équivalence. Or, comme ce mot fut introduit dans le dictionnaire français comme «Pacha», nous avons opté pour le maintien du mot " Effendi" emprunté au turc pour conserver le plus grand nombre de culturèmes.

\section{- Exemple (3): سردار الجيث}

\begin{tabular}{|c|c|c|}
\hline Notre traduction & $\begin{array}{c}\text { Traduction proposée par } \\
\text { les étudiants }\end{array}$ & Texte source \\
\hline $\begin{array}{c}\text { Sirdar (commandant en } \\
\text { chef) de l'armée) }\end{array}$ & $\begin{array}{l}\text { Commandant en chef de } \\
\text { l'armée }\end{array}$ & سردار الجيث \\
\hline
\end{tabular}

Sirdar ou Serdar: subst. masc. : [Sous la forme serdar] Chef militaire dans l'Empire ottoman et en Perse. [Sous la forme sirdar] Officier général anglais qui, en Égypte, commandait l'armée

${ }^{93}$ Le trésor de la langue française informatisé(TLFi),

http://atilf.atilf.fr/dendien/scripts/tlfiv5/advanced.exe?8;s=735364155;

Dernière consultation le 19 mai 2018

${ }^{94}$ Dictionnaire Almaany en ligne, www.almaany.com Dernière consultation le 19 mai 2018.

$$
9595
$$


khédivale. Emprunté au turc serdar «général en chef », et celui-ci au persan « chef, commandant » $(\text { sar «tête », dar « qui possède » })^{96}$.

Pour traduire le mot arabe سردار les étudiants avaient recouru à l'équivalence culturelle et l'avaient traduit en «commandant en chef ». Cependant et comme le montre l'exemple ci- dessus, nous avons proposé aux étudiants d'utiliser un double procédé de traduction : l'emprunt et l'équivalence. De cette façon, nous avons pu à la fois maintenir l'appellation originale tout en donnant au lecteur l'équivalent dans sa culture.

\subsection{Problèmes syntaxiques :}

En matière de traduction, le problème qui se pose toujours devant le traducteur est celui de savoir dans quelle mesure il peut ou doit conserver les structures syntaxiques lors du passage de la languesource à la langue-cible. Chaque système possédant ses propres règles, les choix structurels ou syntaxiques de l'auteur original ne pourront être systématiquement conservés, et ce pour plusieurs raisons : tout d'abord, lorsqu'une structure syntaxique n'a pas d'équivalent direct dans la langue-cible, le traducteur se trouve a fortiori contraint à en choisir une autre structure afin de véhiculer le même contenu informationnel.

De plus, le maintien des structures peut aboutir parfois à des maladresses, lourdeurs ou approximations stylistiques : le traducteur devrait alors réorganiser la phrase afin de rendre celle-ci non seulement correcte mais aussi fluide. Également, il se peut qu'une structure syntaxique ne parvienne pas à transmettre correctement le sens véhiculé dans la structure de départ. Le traducteur devrait alors s'orienter vers une autre structure capable de véhiculer plus fidèlement le sens approprié.

Parmi les problèmes d'ordre structural rencontrés dans notre corpus, nous avons relevé un problème majeur au niveau des propositions : celui de la juxtaposition.

${ }^{96}$ Le Trésor de la Langue Française informatisé(TLFi), http://atilf.atilf.fr/dendien/scripts/tlfiv5/advanced.exe?8; $=735364155$;

Dernière consultation le 19 mai 2019. 


\subsubsection{Juxtaposition des propositions:}

La langue arabe a tendance à juxtaposer plusieurs propositions à l'intérieur d'une même phrase, on parle alors de " phrase / paragraphe". En revanche, la langue française préfère la subordination et les pauses afin de marquer les moments essentiels ou l'apparition de nouvelles données. Les étudiants, de peur de changer la structure de la phrase arabe, ont maintenu la même longueur des phrases. Cette juxtaposition des propositions à l'intérieur d'une même phrase, comme dans les exemples ci-dessus, conduit, dans la langue cible, à un style affaibli et relâché où l'événement clé peut se perdre à l'intérieur d'une multiplicité de propositions juxtaposées et passer inaperçu . Pour surmonter ce problème, nous avons proposé aux étudiants de découper la longue phrase arabe en plus petites en français et ce, d'après l'unité de sens.

- Exemple ( 1 ):

La phrase et sa traduction avant l'alignement :

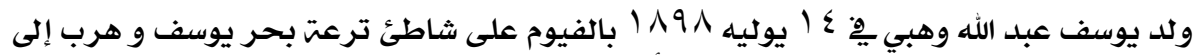

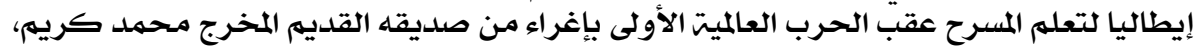

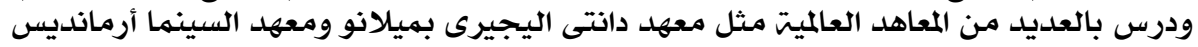

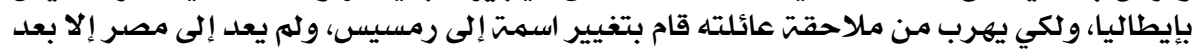

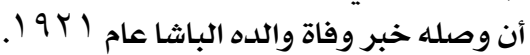

Youssef Abdallah Wahby est né le 14 juillet 1989 au Fayoum au bord du canal de Bahr Youssef (Canal de Joseph). Après la première guerre mondiale, encouragé par son ancien ami le réalisateur Mohamed Koraim, il s'enfuit vers l'Italie. Là-bas, il étudie le thêâtre dans plusieurs instituts internationaux, notamment l'Institut Dante Alighieri à Milan et l'Institut du cinéma Armandis en Italie. Afin d'échapper à la poursuite de sa famille, il change son nom en Ramsès. Il n'est rentré en Égypte qu'en 1921 lorsque lui parvint la nouvelle du décès de son père, le Pacha. 
La phrase et sa traduction après l'alignement du découpage:

\begin{tabular}{|c|c|}
\hline $\begin{array}{l}\text { Youssef Abdallah Wahby est né le } 14 \\
\text { juillet } 1989 \text { au Fayoum au bord du canal } \\
\text { de Bahr Youssef (Canal de Joseph) // }\end{array}$ & 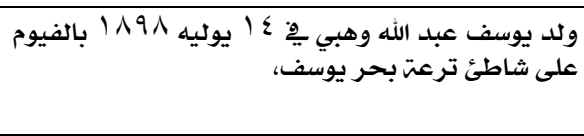 \\
\hline $\begin{array}{l}\text { Après la première guerre mondiale, } \\
\text { encouragé par son ancien ami le } \\
\text { réalisateur Mohamed Koraim, il s'enfuit } \\
\text { vers l'Italie// }\end{array}$ & والاو هرب بإغراء إيطن صداليا لتعلم المديمرح عقب المخرج محمد كريم، العالميت \\
\hline $\begin{array}{l}\text { Là-bas, il étudie le théâtre dans plusieurs } \\
\text { instituts internationaux., notamment } \\
\text { l'Institut Dante Alighieri à Milan et } \\
\text { l'Institut du cinéma Armandis en Italie.// }\end{array}$ & واليجيرى بميلانيو ومعهد المعينما أرمانديس مثل معايطاليا، دانتى \\
\hline $\begin{array}{l}\text { Afin d'échapper à la poursuite de sa } \\
\text { famille, il change son nom en Ramsès.// }\end{array}$ & ولكي يهرب من ملاحقت عائلته قام بتغيير اسمت إلى \\
\hline \multirow{2}{*}{$\begin{array}{l}\text { II n'est rentré en Égypte qu'en } 1921 \\
\text { lorsque lui parvint la nouvelle du décès } \\
\text { de son père, le Pacha. }\end{array}$} & ولبه يعد إلى مصر إلا بعد أن وصله خبر وفاة والده \\
\hline & \\
\hline
\end{tabular}

Ainsi dans cette première phrase, la longue phrase arabe a été divisée en cinq phrases françaises.

- Exemple ( 2 ):

La phrase et sa traduction avant l'alignement:

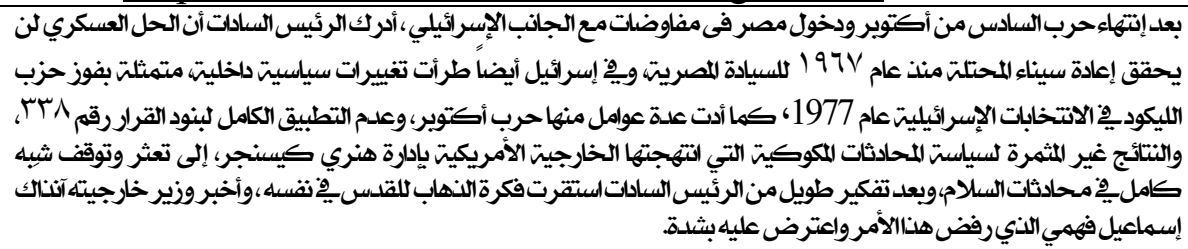

«Après la guerre de 1973, suivie par des négociations entre l’Égypte et Israël, le président Sādāte a compris qu'une solution militaire ne restituera pas le Sinaï, occupée depuis 1967, à la souveraineté égyptienne. De même, il y avait eu des changements intervenus en Israël, telle la victoire du parti du Likoud aux élections israéliennes en 1977. De plus , la guerre de 1973, l'application non intégrale des termes de la Résolution no.338, aussi que les résultats fructueux de la politique des entretiens indirects adoptés par le Département d'État américain sous la direction d'Henry Kissinger, ont mené au blocage et à la perturbation presque totale des pourparlers de paix. Après une longue réflexion, le Président Sādāte a décidé de se rendre luimême à Jérusalem, et a passé la nouvelle à Ismail Fahmī, le ministre des affaires étrangères à l'époque, qui a protesté fortement contre cette décision et l'a refusée catégoriquement » 
La phrase et sa traduction après l'alignement du découpage :

\begin{tabular}{|c|c|}
\hline $\begin{array}{l}\text { Après la guerre de 1973, suivie par des } \\
\text { négociations entre l'Égypte et Israël, le président } \\
\text { Sādāte a compris qu'une solution militaire ne } \\
\text { restituera pas le Sinaï, occupée depuis 1967, à la } \\
\text { souveraineté égyptienne.// }\end{array}$ & 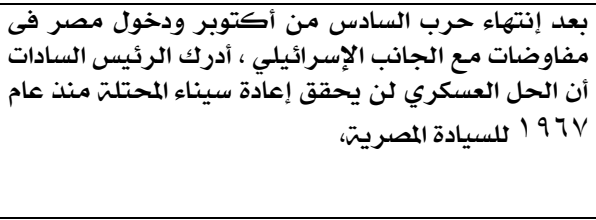 \\
\hline $\begin{array}{l}\text { De même, il y avait eu des changements } \\
\text { intervenus en Israël, telle la victoire du parti du } \\
\text { Likoud auxélections israéliennes en 1977.// }\end{array}$ & 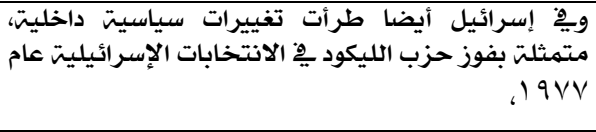 \\
\hline $\begin{array}{l}\text { De plus, la guerre de } 1973 \text {, l'application non } \\
\text { intégrale des termes de la Résolution no.338, aussi } \\
\text { que les résultats fructueux de la politique des } \\
\text { entretiens indirects adoptés par le Département } \\
\text { d'État américain sous la direction d'Henry } \\
\text { Kissinger, ont menéau blocage età la perturbation } \\
\text { presque totale des pourparlers de paix.// }\end{array}$ & 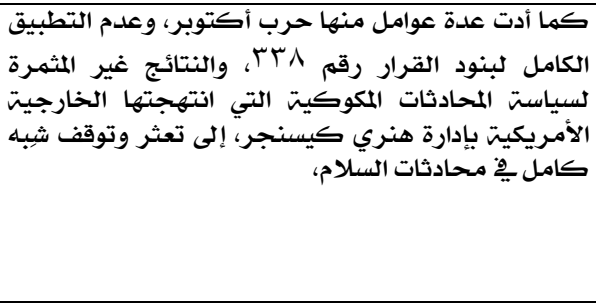 \\
\hline $\begin{array}{l}\text { Après une longue réflexion, le Président Sādāte a } \\
\text { décidé de se rendre lui- même à Jérusalem, et a } \\
\text { passé la nouvelle à Ismail Fahmī, le ministre des } \\
\text { affaires étrangères à l'époque, qui a protesté } \\
\text { fortement contre cette décision et la refusée }\end{array}$ & 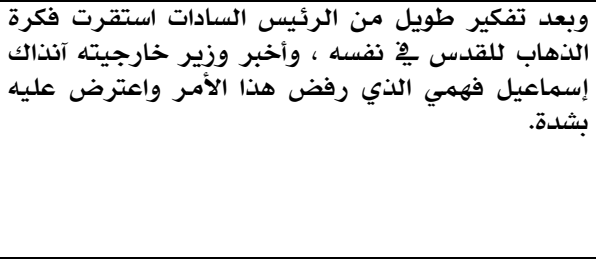 \\
\hline
\end{tabular}

Ainsi, dans ce second exemple, la longue phrase arabe a été divisée en quatre phrases françaises.

\section{- Conclusion :}

En guise de conclusion, nous espérons, par ces quelques réflexions et ces exemples tirés du travail des étudiants de la Filière des Langues Appliquées sur le projet de traduction vers le français du site de la Bibliothèque d'Alexandrie La Mémoire de l'Egypte Moderne, avoir jeté un peu de lumière sur les types d'erreurs que peut rencontrer un traducteur de textes appartenant au Patrimoine culturel et historique d'un pays ou d'une Nation. Dans ce projet entrepris avec les étudiants, nous avons essayé d'aller plus loin et de leur expliquer qu'il ne suffit pas de reconnaître les mots pour saisir le sens d'un énoncé mais que pour arriver à un tel résultat, il faut réaliser la concordance fructueuse du bagage cognitif du lecteur-traducteur avec ses capacités linguistiques. 
Dans le cadre de cette approche, le sens n'est pas conçu comme une entité statique qui est à extraire du texte, mais plutôt comme une entité dynamique qui prend forme au cours de l'effort du traducteur de détecter et d'appréhender au niveau cognitif son contenu, pour ensuite le reformuler dans le texte d'arrivée. Ainsi, les étapes du processus de traduction (compréhension du texte de départ, médiation culturelle et linguistique, mise en forme du texte d'arrivée) ne sont plus considérées comme une simple séquence de décodage et de recodage de messages, mais comme des processus complexes d'inférence et de restructuration $^{97}$.

Aussi, nous leur avions montré que, pour comprendre des phrases ou des notions d'une certaine opacité, il fallait obligatoirement effectuer des opérations de recherche, d'analyse et de décomposition d'éléments linguistiques complexes en unités plus simples, pour pouvoir les représenter et les traduire de la façon la plus correcte. Sur le plan pédagogique, nous avons voulu rendre plus explicites les notions traditionnelles de faux sens, de non-sens ou d'ambiguïté rencontrées par les étudiants, en remontant avec eux à la source de l'erreur, en les discutant avec eux et en leur offrant des outils pour en mesurer la gravité afin qu'ils puissent acquérir une meilleure conscience de leurs lacunes et en trouver les solutions appropriées.

Dans ce travail qui s'est avéré très enrichissant et pour les étudiants et pour nous en tant qu'enseignante de traduction, nous avons essayé, en plus, de leur prouver que les erreurs ou les impropriétés commises par les traducteurs de textes dits anciens ou de textes relatant le patrimoine propre à une Nation proviennent surtout du manque de culture dont souffrent maints traducteurs modernes Cela signifie qu'il arrive que les auteurs de ces textes spécifiques parlent de réalités qui leur étaient ordinaires ou relativement familières et qui, à ce titre, paraissaient ne leur réclamer aucune explication, quoique sommaire. Or, avec le temps, certaines de ces réalités, ne deviennent ni familières ni ordinaires; et par conséquent, causent de vrais problèmes pour un bon nombre de traducteurs modernes. Le cas est encore plus grave s'il s'agit d'étudiants en phase de préparation pour devenir des futurs traducteurs. .

${ }^{97}$ Cf. Périclès PAPAVASSILIOU: « Traductologie et sciences cognitives : une dialectique prometteuse », in M3ta Volume 52, Numéro 1, mars 2007, pp. 29-36 
Il arrive aussi qu'une traduction faite à une époque pourrait tomber en disgrâce aux siècles suivants. Le rôle du traducteur est ici d'une importance majeure, car il doit rendre claires et compréhensibles des notions traduites à une époque mais qui deviennent obscures à une époque plus moderne en raison de l'absence du référent culturel de ces notions. Le rôle du traducteur est ici comparable à un couturier ou tailleur qui donne une nouvelle allure à un vêtement démodé.

A la fin de ce projet, nos étudiants ont été convaincus qu'un bon traducteur doit compenser par une profonde culture, les décalages qu'imposent à leurs lecteurs les textes comprenant des culturèmes, ou autrement dit, des notions culturelles ou historiques anciennes, révolues ou différentes de la culture cible. Et ils sont parvenus par eux-mêmes à réaliser ce que Jean René Ladmiral avait souligné, quelques années plus tôt, que l'acte de traduction est, tout d'abord, une activité de compréhension du texte premier, ou du texte de départ, qu'il est ensuite une activité de transfert puisqu'il permet le passage d'un système culturel, linguistique et rhétorique du français vers un autre système tout à fait différent ; qu'il est en plus une activité de rédaction et qu'il est enfin une activité de révision ou de relecture. Il ajoute aussi que l'acte de traduction est surtout, une activité de recherche documentaire ${ }^{98}$.

Bref, le point d'orgue du travail du traducteur réside donc dans « sa compréhension », considérée par la plupart des théoriciens et des enseignants de la traduction comme la condition sine qua non de la traduction. Comprendre et «digérer », si nous osons le dire, le texte de départ peut épargner au traducteur tous les types d'impropriétés et d'ambiguïtés qui pourraient mener à des conséquences désastreuses. Un traducteur doit donc réussir à faire passer, dans le texte d'arrivée, ce qu'il comprend et «savoure» du texte de départ car les véritables erreurs ou impropriétés dans un texte traduit naissent, très souvent, de situations où le traducteur ne comprend pas ou comprend mal le message qu'il doit traduire.

Nous espérons vraiment, à l'issue de cette étude, avoir pu répondre aux questions formulées dans l'introduction. Nous souhaitons surtout que ce projet voie le jour et que le site de la bibliothèque La Mémoire

${ }^{98}$ Cf. J.R. LADMIRAL, Théorèmes pour la traduction, Payot, Paris 1979, p.19. 
de l'Egypte Moderne traduit par les étudiants de la Filière des Langues Appliquées dans le cadre de leur projet de fin d'études en Traduction et révisé, une première fois, par un groupe d'enseignants dont nous faisons partie, soit une pierre ajoutée à l'édifice de la traduction du Patrimoine égyptien ! 


\section{Bibliographie}

\section{I- Corpus :}

«Mémoire de l'Égypte Moderne » (MoME) ذكرة مصر الحديثة/

http://modernegypt.bibalex.org/collections/home/default.aspx

\section{II- Ouvrages consultés :}

AMBROSETTI, Elena: Egypte, l'exception démographique, Coll. Les Cahiers de l'INED, éds. de l'INED.

BAUER, G. Namenkunde des Deuschen, Germanistische Lehrbuchsammlung Band, 1985.

DUBOIS, G. Dictionnaire de linguistique, Paris, Larousse, 1973.

GHAZALA, Hasan: Translation as Problems and Solutions: course-book for university students and trainee translators. Dar Al-alam, Beyrouth, 1995.

GOUADEC, Daniel Traduire - Stratégies de la traduction entre l'anglais et le français - Essai de définition d'un modèle de formation des traducteurs - Métiers des langues/Langues des Métiers - La Maison du Dictionnaire,2001.

GUIDÈRE, Mathieu : Introduction à la traductologie, De Boeck, Bruxelles, 2008.

HAMONT, Pierre Nicolas : L'Egypte, sous Méhémet-Ali, Léautey et Lecointe, Paris, 1843.

JONASSON, K. Le nom propre. Constructions et interprétations, Duculot, Paris, 1994.

LADMIRAL, J.R., Théorèmes pour la traduction, Payot, Paris 1979.

LEDERER, Marianne : La traduction aujourd'hui. Le modèle interprétatif, Hachette Paris, 1994.

LUQUET, Gilles: Travaux de linguistique hispanique, Presses de la Sorbonne Nouvelle, 1998.

POLGUERE, A. : Lexicologie et sémantique lexicale. Notions fondamentales, Presses de l'Université de Montréal, 2003.

SELESKOVITCH, Dancia et LEDERER, Marianne: Interpréter pour traduire, Didier érudition, Paris, 1984. 
- VINAY, J.-P. et DARBELNET: J., Stylistique comparée du Français et de l'Anglais, Didier, 2004.

\section{III- Articles consultés :}

AGAFONOV, Claire, GRASS et al, Thierry. : « La traduction multilingue des noms propres dans PROLEX », in M3TA, Volume 51, numéro 4, décembre 2006, p.327.

CRISTINOI-BURSUC, Antonia: «Les erreurs dans la traduction automatique du genre dans les couples françaisanglais et anglais-français : typologie, causes linguistiques et solutions » in Revue française de linguistique appliquée, 2009/1 (Vol. XIV), pages 93 à 108.

DAILLE, Béatrice, FOUROUR, Nordine \& MORIN, Emmanuel : «Catégorisation des noms propres : une étude en corpus », in Cahiers de Grammaire 25 (2000), «Sémantique et Corpus », pp. 115-129.

DANCETTE, Jeanne «La faute de sens en traduction », in TTR (Traduction, terminologie, rédaction) , Volume 2, numéro 2, 2e semestre 1989, p.83.

DURIEUX, Christine : « Mettre la main sur le figement lexical : la démarche du traducteur», in Meta, 53, 2008, p. 324.

GROSS, Maurice: "Sur les phrases figées complexes du français », in Langue française 77, pp. 47-70.

- MEJRI, Salah :« Figement et traduction : problématique générale », in M3TA, 53, 2008, p. 245.

- PAPAVASSILIOU, Périclès: « Traductologie et sciences cognitives : une dialectique prometteuse », in M3TA, Volume 52, Numéro 1, mars 2007, pp. 29-36

- RICHARD, Marc: «Des dénominations multiples de lieux dans la perspective de la normalisation des noms géographiques » Working Paper in Commission de toponymie du Québec, n.17, 16 mai 1989, pp.1-19.

- SAAD ALI, Mohamed: "La traduction des expressions figées : langue et culture", in Traduire, 235 | 2016, pp. 103-123.

- TUTIN, Agnès : «Les collocations lexicales : une relation essentiellement binaire définie par la relation prédicatargument », in Langages , $\mathrm{n}^{\circ} 189,2013$, pp. 47-63. 
- TUTIN Agnès, GROSSMANN, Francis : «Collocations régulières et irrégulières : esquisse de typologie du phénomène collocatif », in Revue française de linguistique appliquée , 2002, Vol. VII, pp. 7 à 25.

\section{I- Ouvrages en ligne :}

\section{Webographie}

LACROUX, Jean-Pierre: Orthotypographie : Orthographe \& typographie françaises : Dictionnaire raisonné, 2007),

http://www.orthotypographie.fr/

Dernière consultation le 03/08/2020

MALEKOU, Paul Edwin : Les anthroponymes et toponymes

Gisir: proposition d'un modèle de dictionnaire, Maîtrise 2006, Université Omar BONGO .

https://www.memoireonline.com/07/08/1412/m_anthroponym es-toponymes-gisir-proposition-modele-dictionnaire 5.html

Dernière consultation le 03/08/2020

\section{II- Articles en ligne :}

BADEA, Georgiana I. : «La traduction de l'écart culturel », in https://www.academia.edu/1249770/La_traduction_de_lecart_ culturel

Dernière consultation le 18/07/2020

GERSAL, Frédérick: «Les anthroponymes nous aident à comprendre l'Histoire», in https://www.lefigaro.fr/languefrancaise/expressions-francaises

/2016/09/21/37003-20160921ARTFIG00063-frederick-gersalles-anthroponymes-nous-aident-a-comprendre-l-histoire.php GUILLEUX, Céline: " Les expressions figées : analyse linguistique et littéraire et exploitation en classe de français langue étrangère (FLE) », in, Calenda, Publié le mercredi 25 juillet 2018.

https://calenda.org/461019

Dernière consultation le 10/8/2020 
LÖFSTRÖM, Jonas, SCHNABEL, Betina : " Comment analyser et comparer les toponymes de différentes langues dans une perspective synchronique ", in Nouvelle revue d'onomastique [en ligne], $\mathrm{n}^{\circ} 52,2010$. pp. 291-318

https://www.persee.fr/doc/onoma_0755-

7752_2010_num_52_1_1549

Dernière consultation le 09/09/2020

MAUREL, Denis et TRAN, Mickaël : «Une ontologie multilingue des noms propres » in Corela

https://doi.org/10.4000/corela.1203

Dernière consultation le 02/05/2019

MILLET, Bertrand: «Genèse de la presse enfantine en Egypte 1870-1915», pp. $25-35$

in https://books.openedition.org/cedej/512?lang=fr\#text Dernière consultation le 19/07/2020

SOLE, Robert : «Fouad Ier :Une monarchie cosmopolite », in https://www.cairn.info/ils-ont-fait-1-egypte-moderne-9782262064235-page-151.htm

Dernière consultation le 02/05/2019

WEBER Nadine: "Titres honorifiques », in https://hls-dhsdss.ch/fr/articles/017435/2013-12-18/

Dernière consultation le 02/05/2019

\section{III- Dictionnaires en ligne :}

Encyclopédie Universalis :

http://www.universalis.fr/encyclopedie/wahhabisme/

Dictionnaire de l'Islam (religion et civilisation)

Dernière consultation le 18/05/2017

Centre National de Ressources Textuelles et Lexicales :

http://www.cnrtl.fr/definition/erreur

Dernière consultation le 08/09/2019

Le Trésor de la Langue Française informatisé :

https://academie.atilf.fr/9/consulter/erreur?page=1

Dernière consultation le 08/09/2019

Dictionnaire Larousse en ligne :

https://www.larousse.fr/dictionnaires/francais/erreur/30846?q=erreur $\# 30759$

Dernière consultation le 08/09/2019 
Dictionnaire Almaany en ligne :

https://www.almaany.com/ar/dict/ar-

ar/\%D8\%B1\%D9\%88\%D8\%B6\%D8\%A9/

Dernière consultation le 19/07/2020

- Dictionnaire Linternaute en ligne

https://www.linternaute.fr/sport/biographie/1777200-

mohamed-ali-biographie-courte-

datescitations/\#: :text=Biographie $\% 20$ courte $\% 20 \mathrm{de} \% 20 \mathrm{Moha}$ med\%20Ali\%20\%2D\%20N\%C3\%A9\%20le\%2019\%20janvier $\% 201942$,reprises\%2C\%20sacr\%C3\%A9\%20champion\%20du $\% 20$ monde

Dernière consultation le 04/08/2020

Dictionnaire Sensagent en ligne :

http://dictionnaire.sensagent.leparisien.fr/en\%20fin\%20de\%20 compte/fr-fr/

Dernière consultation le 08/09/2020

Dictionnaire Almoheet en ligne

https://almoheet.net/\%D8\%B4\%D8\%B9\%D8\%B1-

$\% \mathrm{D} 8 \% \mathrm{~B} 9 \% \mathrm{D} 8 \% \mathrm{~A} 8 \% \mathrm{D} 8 \% \mathrm{AF}-$

\%D8\%A7\%D9\%84\%D8\%B9\%D8\%B2\%D9\%8A\%D8\%B2-

\%D8\%A7\%D9\%84\%D9\%85\%D9\%82\%D8\%A7\%D9\%84\%D

$8 \% \mathrm{AD} /$

Dernière consultation le 08/09/2020

- Dictionnaire Almoslim en ligne

https://almoslim.net/node/241806

Dernière consultation le 08/09/2020

\section{IV-Sites :}

https://www.les-treilles.com/les-intraduisibles-du-patrimoine

Dernière consultation le 20/09/ 2018.

- $\quad$ https://www.dea.univr.it/documenti/OccorrenzaIns/matdid/mat did189516.pdf

Dernière consultation le 21/09/ 2018.

https://www.cairn.info/revue-defense-nationale-2015-10-page-

$\underline{122 . h t m}$

Dernière consultation le 05/03/2019 
https://www.france-education-international.fr/article/le-

systeme-educatif-francais?langue $=\mathrm{fr}$

Dernière consultation le 05/05/2019

- https://gate.ahram.org.eg/daily/News/131747/121/458256/\%D 8\%AA\%D8\%B1\%D8\%A7\%D8\%AB-\%D9\%88\%D8\%AD\%D8\%B6\%D8\%A7\%D8\%B1\%D8\%A9/\%D8\%AD \%D9\%83\%D8\%A7\%D9\%8A\%D8\%A9\%D8\%AF\%D8\%A7\%D8\%B1\%D8\%A7\%D9\%84\%D8\%B9\%D9\%84\%D9\%80\%D9\%80\%D 9\%88\%D9\%85.aspx

Dernière consultation le 03/05/2019

- https://www.amazon.fr/trilogie-Chehayed-CommentairesPhilippe-Traduction/dp/B015YMH8M6

Dernière consultation le 20/09/2019.

- http://collections.quaibranly.fr/?query=objectnumber=[PV008 8174]\&showtype=record\#350b4061-96fe-419e-ae5917 fa4d3b675a

- https://www.lepoint.fr/societe/le-grand-imam-d-al-azhar-lhomme-qui-detient-les-cles-de-l-islam-17-06-20162047402_23.php Dernière consultation le 19/05/ 2019.

- https://www.bfmtv.com/societe/la-valse-des-mots-disparusdes-dictionnaires_AV-201811150079.html

Dernière consultation le 18/07/ 2019

- https://www.btb.termiumplus.gc.ca/tpv2guides/guides/chroniq/ index-

fra.html?lang=fra\&lettr=indx_autr8KQ_bboMdGvY\&page=9 X0H4FN_ZoEM.html\#note4 Dernière consultation le $06 / 02 / 2019$

- https://beelingwa.com/fr/blog/erreur-de-traduction-10exemples Dernière consultation le 06/02 2019

- http://www.esa.gov.eg/history.aspx Dernière consultation le 06/06/2019

- http://www.dadc.gouv.tg/index.php/direction/visionmissions.html Dernière consultation le 06/06/2019 
- https://www.france-education-international.fr/article/lesysteme-educatif-francais?langue $=\mathrm{fr}$ Dernière consultation le 05/05/2019

- $\quad$ https://al-maktaba.org/book/31917/169

Dernière consultation le 05/03/2019

- https://jrayed.org/ar/newspapers/falastin/1911/09/02/01/article/ 2/?e=--------ar-20--1--img-txIN\%7ctxTI--------------1

Dernière consultation le 09/05/2019

- http://www.culture.fr/eng/collections/resultats/(offset)/140/(pa ge count) $/ 2333$ ? keywords=barrage Dernière consultation le 20/09/ 2020. https://www.marefa.org/\%D8\%A7\%D9\%84\%D8\%B4\%D8\% B1\%D9\%8A\%D8\%AF_(\%D9\%81\%D9\%8A\%D9\%84\%D9\% $\underline{85)}$

Dernière consultation le 19/07/2020

- https://hebdo.ahram.org.eg/NewsContent/1011/32/97/5046/Al Qanater,-un-mod\%C3\%A8le-dirrigation-moderne.aspx Dernière consultation le 09/11/2020

- https://www.sis.gov.eg/Story/84113/Al-Qanater-AlKha\%C3\%AFriya?lang=fr Dernière consultation le 09/11/2020

- $\quad$ http://bdl.oqlf.gouv.qc.ca/bdl/gabarit_bdl.asp?id=4271 Dernière consultation le 04/08/2020 